\title{
MATHEMATICAL MODELING OF FLUORESCENCE DIFFUSE OPTICAL IMAGING OF CELL MEMBRANE POTENTIAL CHANGES
}

BY

HABIB AMMARI (Department of Mathematics and Applications, Ecole Normale Supérieure, 45 Rue d'Ulm, 75005 Paris, France),

JOSSELIN GARNIER (Laboratoire de Probabilités et Modèles Aléatoires E Laboratoire Jacques-Louis Lions, Université Paris VII, 75205 Paris Cedex 13, France),

AND

LAURE GIOVANGIGLI (Department of Mathematics and Applications, Ecole Normale Supérieure, 45 Rue d'Ulm, 75005 Paris, France)

\begin{abstract}
The aim of this paper is to provide a mathematical model for spatial distribution of membrane electrical potential changes by fluorescence diffuse optical tomography. We derive the resolving power of the imaging method in the presence of measurement noise. The proposed mathematical model can be used for cell membrane tracking with the resolution of the optical microscope.
\end{abstract}

1. Introduction. The propagation of light through a highly scattering medium with low absorption is well described by the diffusion equation [30. Diffuse optical imaging techniques measure the spatially-dependent absorption and scattering properties of a tissue. A light source illuminates the tissue, and detectors measure the intensity of the exiting light at the boundary of the tissue after it undergoes multiple scattering and absorption. One can use these measurements to reconstruct, from the diffusion equation, a map of the optical parameters of the studied biological tissue [25, 38].

Diffuse optical imaging techniques use near infrared light, because absorption by biological tissue is minimal at these wavelengths, and one can then produce images deep in living subjects or samples, up to several centimeters.

These techniques can be used to image fluorescing targets, known as fluorophores, in tissues. When excited by light at a specific wavelength, fluorophores emit light at

Received April 13, 2012.

2010 Mathematics Subject Classification. Primary 35R30, 35B30.

Key words and phrases. Resolving power, stability and resolution analysis, fluorescence diffuse optical tomography, cell tomography, cell membrane, electric field, layer potential techniques.

This work was supported by ERC Advanced Grant Project MULTIMOD-267184.

E-mail address: habib.ammari@ens.fr

E-mail address: garnier@math.jussieu.fr

E-mail address: laure.giovangigli@ens.fr 
a different wavelength in order to decay to their ground state. Measurements of emitted light exiting at the boundary of the tissue, combined with measurements of residual excitation light from sources after it went through the tissue provide an insight of the tissue optical properties. More precisely, these measurements allow us to reconstruct a map of the tissue optical parameters, the distribution of fluorophore concentration, and fluorophore lifetime, which is the time they spend in their excited state before emitting light [13, 31]. The fluorescent indicators, which can be chosen with excitation and emission wavelengths in the near infrared light spectrum, accumulate in specific areas. With such techniques, one can then localize proteins, cells or diseased tissues, visualize in vivo biological processes, and obtain measurements of the concentration in tissues of important physiological markers, such as oxygenated hemoglobin [28, 29, 40]. Detailed structural information as well as indications of pathology can be obtained from these images.

In this paper, we mathematically formulate the imaging problem of the spatial distributions of the transmembrane potential changes induced in cells by applied external electric fields. The use of optical detection methods for the measurement of fluorescence response to membrane electric fields was reported in the early 1970s. Since then, considerable advances have been reported [23. In [18], it has been demonstrated experimentally that membrane potential changes can be imaged with the resolution of the optical microscopy. The key feature of this system is the combined use of an external electric field and fluorescence tomography. The fluorescent indicators are designed in such a way that they respond linearly to the electrical potential jump across the membrane. The application of the electric field enhances the membrane fluorescence imaging.

The purpose of this paper is threefold. We first provide and analyze a mathematical model for optical imaging of changes in membrane electric potentials. Then we propose, in the linearized case where the shape of the cell is a perturbation of a disk, an efficient direct imaging technique based on an appropriate choice of the applied currents. An iterative imaging algorithm for more complex shapes is also suggested. Finally, we estimate the resolving power of the proposed imaging algorithm in the presence of measurement noise. In a forthcoming work, we will use the proposed algorithm for implementing tracking approaches capable of imaging the behavior of single or cluttered live cells.

Our main results in this paper can be summarized as follows. Let $C$ be the cell, and let $\Omega$ be the background domain. Given an optical excitation $g$, the emitted light fluence is $\Phi_{\mathrm{emt}}^{g}$, the solution to the diffusion equation (2.3) with $\Phi_{\mathrm{exc}}^{g}$ defined by (2.2) and $c_{\mathrm{flr}}$ being the concentration of fluorophore supported on the cell membrane $\partial C$. Equation (2.5) gives the relation between the function $c_{\mathrm{ffr}}$ and the electric potential $u$ defined by (2.4). In order to image the cell membrane $\partial C$, we establish identity (4.1) and linearize in Theorem 4.11 relation (2.5) for $\partial C$ being a perturbation of a disk. Proposition 4.12 gives the least squares estimate of the cell membrane perturbation. Introducing the signal-tonoise ratio in (4.55), where $\sigma$ models the measurement noise amplitude and $\epsilon$ corresponds to the order of magnitude of the cell membrane perturbation, we derive in Theorem 4.13 the resolving power of the imaging method. Theorem 4.14 which is our main result in this paper, provides expressions for the reconstructed modes in the cell membrane perturbation in the presence of measurement noise under physical assumptions on the 
size of the cell and the value of the used frequency. A generalization of the linearization procedure for arbitrarily shaped cell membranes is provided in Proposition 4.15, and the reconstruction of perturbations of arbitrarily shaped cell membranes is formulated as a minimization problem, where the data is appropriately chosen in order to maximize the resolution of the reconstructed images.

2. Governing model. We consider a cell that we want to image. We inject fluorescent indicators that stick only on the cell membrane [26]. These markers are chosen so that their concentration responds linearly to the potential jump across the membrane, when the cell is immersed in an external electric field [18. We apply such an external electric field at the boundary of our domain and use fluorescence optical diffuse tomography to reconstruct the position and shape of the membrane.

2.1. Coupled diffusion equations. A sinusoidally modulated near infrared monochromatic light source $g$, located at the boundary $\partial \Omega$ of the examined domain $\Omega$, launches an excitation light fluence

$$
\phi_{\mathrm{exc}}=\Phi_{\mathrm{exc}}(x, \omega) e^{i \omega t}
$$

at the wavelength $\lambda_{\text {exc }}$ into $\Omega$. At time $t$ and point $x, \phi_{\text {exc }}$ represents the average photon density due to excitation by the source oscillating at frequency $\omega$. After it undergoes multiple scattering and absorption, this light wave reaches the fluorescent markers that are accumulated on $\partial C$, the membrane of the cell $C$. The excited fluorophores emit a wave

$$
\phi_{\mathrm{emt}}=\Phi_{\mathrm{emt}}(x, \omega) e^{i \omega t}
$$

at the wavelength $\lambda_{\text {emt }}$. The intensity of the emitted wave is proportional to the intensity of the excitation wave when it reaches the fluorescent molecule. The emitted waves pass through the absorbing and scattering domains and are detected at the boundary $\partial \Omega$.

In the near infrared spectral window, the propagation of light in biological tissues can be modeled by the diffusion equation, which is a limit of the radiative transport equation when the transport mean free path is much smaller than the typical propagation distance. Our model can therefore be described by the following coupled diffusion equations completed by Robin boundary conditions [19, 31, 36, 37]:

$$
\begin{gathered}
\left\{\begin{array}{cc}
-\nabla \cdot\left(D_{\mathrm{exc}}(x) \nabla \Phi_{\mathrm{exc}}(x, \omega)\right)+\left(\mu_{\mathrm{exc}}(x)+\frac{i \omega}{c}\right) \Phi_{\mathrm{exc}}(x, \omega)=0 & \text { in } \Omega, \\
\ell_{\mathrm{exc}} \frac{\partial \Phi_{\mathrm{exc}}}{\partial \nu}(x, \omega)+\Phi_{\mathrm{exc}}(x, \omega)=g(x) & \text { on } \partial \Omega, \\
=\gamma(x, \omega) \Phi_{\mathrm{exc}}(x, \omega) \quad \text { in } \Omega, \\
-\nabla \cdot\left(D_{\mathrm{emt}}(x) \nabla \Phi_{\mathrm{emt}}(x, \omega)\right)+\left(\mu_{\mathrm{emt}}(x)+\frac{i \omega}{c}\right) \Phi_{\mathrm{emt}}(x, \omega) \\
\ell_{\mathrm{emt}} \frac{\partial \Phi_{\mathrm{emt}}}{\partial \nu}(x, \omega)+\Phi_{\mathrm{emt}}(x, \omega)=0 \quad \text { on } \partial \Omega
\end{array}\right.
\end{gathered}
$$

Here,

- $\nu$ denotes the outward normal at the boundary $\partial \Omega$;

- $c$ denotes the speed of light in the medium; 
- $D_{\text {exc }}$ and $\mu_{\text {exc }}$ (respectively $D_{\text {emt }}$ and $\mu_{\text {emt }}$ ) denote the photon diffusion and absorption coefficient at wavelength $\lambda_{\text {exc }}$ (respectively $\lambda_{\text {emt }}$ ) over the speed of light $c$. Assuming that the scattering is isotropic, they can be expressed, for $i=$ exc, emt, as follows:

$D_{i}(x)=\frac{1}{d\left(\mu_{a, i}(x)+\mu_{\mathrm{fr}, i}(x)+\mu_{s, i}^{\prime}(x)\right)} \quad$ and $\quad \mu_{i}(x)=\mu_{a, i}(x)+\mu_{\mathrm{fl}, i}(x)$,

where

- $\mu_{a, i}$ denotes the absorption coefficient, due to natural chromophores of the medium, at wavelength $\lambda_{i}$;

- $\mu_{\mathrm{ffr}, i}$ denotes the absorption coefficient, due to fluorophores, at wavelength $\lambda_{i}$. This absorption coefficient is proportional to the fluorophore concentration $c_{\mathrm{fr}}(x)$. The proportionality coefficient, $\varepsilon_{\text {exc }}$, is the fluorophore extinction coefficient at wavelength $\lambda_{i}$;

- $\mu_{s, i}^{\prime}$ denotes the reduced scattering coefficient at wavelength $\lambda_{i}$; its inverse is the transport mean free path.

$-\ell_{i}$ is the extrapolation length. It is computed from the radiative transport theory [35] and is proportional to the transport mean path. The multiplicative function depends on the index mismatch between the scattering medium in $\Omega$ and the surroundings.

$-d$ is the space dimension;

- $\gamma$ is given by

$$
\gamma(x, \omega)=\frac{\eta \mu_{\mathrm{fr}, \mathrm{exc}}(x)}{1-i \omega \tau(x)}=\frac{\eta \varepsilon_{\mathrm{exc}} c_{\mathrm{fr}}(x)}{1-i \omega \tau(x)},
$$

with $\eta$ and $\tau$ being respectively the fluorophore's quantum efficiency and fluorescence lifetime.

2.2. Model assumptions. Let $\Omega$ be the background domain, and let $C \Subset \Omega$ denote the cell. From now on, the space dimension $d$ is equal to 2 or 3 , and $\Omega$ and $C$ are bounded $\mathcal{C}^{2}$ - domains.

The fluorophores are only located on the cell membrane $\partial C$; their concentration $c_{\mathrm{flr}}(x)$ is zero, except on $\partial C$. We neglect their contribution to the absorption and diffusion coefficient, that is,

$$
D_{i}(x)=\frac{1}{d\left(\mu_{a, i}(x)+\mu_{s, i}^{\prime}(x)\right)} \quad \text { and } \quad \mu_{i}(x)=\mu_{a, i}(x) .
$$

In the near infrared spectral window, the absorption coefficient is much smaller than the reduced scattering coefficient. This is one of the conditions to approximate the light propagation in the medium by the diffusion equation.

We can approximate the diffusion coefficients at the excitation and emission wavelength as follows:

$$
D_{i}(x)=\frac{1}{d \mu_{s, i}^{\prime}(x)} .
$$


We consider that the optical parameters are constant in the domain $\Omega$ and do not depend on the wavelength of the propagating light. Hence, for $i=$ exc, emt,

$$
D_{i}(x)=D_{i}=D=\frac{1}{d \mu_{s}^{\prime}}, \quad \mu_{i}(x)=\mu_{i}=\mu=\mu_{a}, \quad \text { and } \quad \ell_{i}(x)=\ell_{i}=\ell .
$$

We consider that the fluorophore's fluorescence lifetime $\tau$ is constant. From (2.1) it follows that $\gamma$ depends on the position $x$ only through $\mu_{\mathrm{fr}}(x)$ and, more specifically, $c_{\mathrm{frr}}(x)$. It can then be written as follows:

$$
\gamma(x, \omega)=\tilde{\gamma}(\omega) c_{\mathrm{frr}}(x) \quad \text { with } \quad \tilde{\gamma}(\omega)=\frac{\eta \varepsilon_{\mathrm{exc}}}{1-i \omega \tau} .
$$

The coupled diffusion equations and their boundary conditions then become

$$
\begin{gathered}
\left\{\begin{array}{lc}
-D \Delta \Phi_{\mathrm{exc}}^{g}(x, \omega)+\left(\mu+\frac{i \omega}{c}\right) \Phi_{\mathrm{exc}}^{g}(x, \omega)=0 & \text { in } \Omega, \\
\ell \frac{\partial \Phi_{\mathrm{exc}}^{g}}{\partial \nu}(x, \omega)+\Phi_{\mathrm{exc}}^{g}(x, \omega)=g(x) & \text { on } \partial \Omega,
\end{array}\right. \\
\left\{\begin{array}{lc}
-D \Delta \Phi_{\mathrm{emt}}^{g}(x, \omega)+\left(\mu+\frac{i \omega}{c}\right) \Phi_{\mathrm{emt}}^{g}(x, \omega)=\tilde{\gamma}(\omega) c_{\mathrm{frr}}(x) \Phi_{\mathrm{exc}}^{g}(x, \omega) & \text { in } \Omega, \\
\ell \frac{\partial \Phi_{\mathrm{emt}}^{g}}{\partial \nu}(x, \omega)+\Phi_{\mathrm{emt}}^{g}(x, \omega)=0 & \text { on } \partial \Omega,
\end{array}\right.
\end{gathered}
$$

where the source $g$ is in $L^{2}(\partial \Omega)$.

2.3. Electrical model of a cell. We apply at the boundary of our domain an electric field $g_{\text {ele }} \in L^{2}(\partial \Omega)$. We consider that $\Omega \backslash \bar{C}$ and $C$ are homogeneous and isotropic media with conductivity 1 . The thickness $\epsilon$ of the cell membrane is supposed to be small. We denote by $\sigma$ the conductivity of the cell membrane. We assume that $\sigma \ll 1$ and $\beta>0$ to be given by $\beta=\sigma^{-1} \epsilon$; see 23 .

We can approximate the voltage potential $u$ within our medium by the unique solution to the following problem [14,21,32, 34]:

$$
\begin{cases}\Delta u=0 & \text { in } C \cup \Omega \backslash \bar{C} \\ \left.\frac{\partial u}{\partial \nu}\right|_{+}-\left.\frac{\partial u}{\partial \nu}\right|_{-}=0 & \text { on } \partial C, \\ \left.u\right|_{+}-\left.u\right|_{-}=\beta \frac{\partial u}{\partial \nu} & \text { on } \partial C \\ \left.\frac{\partial u}{\partial \nu}\right|_{\partial \Omega}=g_{\text {ele }}, \quad \int_{\partial \Omega} u=0 .\end{cases}
$$

Since we have chosen the fluorescent indicators of the cell membrane such that they respond linearly to the potential jump across the membrane [18, we can express their concentration as

$$
c_{\mathrm{flr}}=\left.\delta[u]\right|_{\partial C},
$$

where $\delta$ is a constant 18 . 
3. Forward problem. The forward problem consists of determining $\left.\Phi_{\text {emt }}\right|_{\partial \Omega}$ for a fixed applied electric field $g_{\text {ele }}$, a light excitation $g$ and a given cell $C$. The optical parameters of the medium, $D$ and $\mu$, the speed of light $c$, the extrapolation length $\ell$ and $\tilde{\gamma}$ are supposed to be known.

3.1. Expression of $\Phi_{\mathrm{exc}}^{g}$. Let $\Phi_{\mathrm{exc}}^{g}$ be the excitation light fluence in $\Omega$, due to an excitation $g$ applied at its boundary $\partial \Omega$. The function $\Phi_{\operatorname{exc}}^{g}$ is the solution to the following problem:

$$
\begin{cases}-\Delta \Phi_{\operatorname{exc}}^{g}(y)+k^{2} \Phi_{\operatorname{exc}}^{g}(y)=0 & \text { in } \Omega, \\ \ell \frac{\partial \Phi_{\operatorname{exc}}^{g}}{\partial \nu}(y)+\Phi_{\operatorname{exc}}^{g}(y)=g & \text { on } \partial \Omega,\end{cases}
$$

where $k^{2}=\frac{\mu+i \omega / c}{D}$. Note that if $\ell=0$, then the Robin boundary condition in (3.1) should be replaced with the Dirichlet boundary condition: $\Phi_{\operatorname{exc}}^{g}(y)=g$ on $\partial \Omega$.

Let $\Gamma$ be the fundamental solution to $-\Delta+k^{2}$. $\Gamma$ is (the exponentially decaying) solution to

$$
\forall y, z \in \mathbb{R}^{d}, \quad-\Delta_{y} \Gamma_{z}(y)+k^{2} \Gamma_{z}(y)=\delta_{z}(y),
$$

where $\delta_{z}$ is the Dirac mass at $z$.

We know the explicit expression of $\Gamma_{z}(y)$ for all $y \neq z \in \mathbb{R}^{d}[\underline{8}$ :

$$
\begin{array}{cc}
\Gamma_{z}(y)=\frac{i}{4} H_{0}^{(1)}(i k|y-z|) & \text { if } d=2, \\
\Gamma_{z}(y)=\frac{e^{-k|y-z|}}{4 \pi|y-z|} & \text { if } d=3,
\end{array}
$$

where $H_{0}^{(1)}$ is the Hankel function of the first kind of order 0 .

We introduce the single and double layer potentials of a function $f \in L^{2}(\partial \Omega)$ for all $z \in \mathbb{R}^{d} \backslash \partial \Omega,[8]$

$$
\begin{gathered}
\forall z \in \mathbb{R}^{d}, \quad \mathcal{S}_{\Omega}[f](z)=\int_{\partial \Omega} \Gamma_{z}(y) f(y) d s(y), \\
\forall z \in \mathbb{R}^{d} \backslash \partial \Omega, \quad \mathcal{D}_{\Omega}[f](z)=\int_{\partial \Omega} \frac{\partial \Gamma_{z}(y)}{\partial \nu} f(y) d s(y) .
\end{gathered}
$$

Lemma 3.1. The double layer potential verifies, for all $f \in L^{2}(\partial \Omega)$,

$$
\begin{array}{cc}
\left(-\Delta+k^{2}\right) \mathcal{D}_{\Omega}[f]=0 & \text { in } \mathbb{R}^{d} \backslash \partial \Omega, \\
\left.\frac{\partial}{\partial \nu} \mathcal{D}_{\Omega}[f]\right|_{+}=\left.\frac{\partial}{\partial \nu} \mathcal{D}_{\Omega}[f]\right|_{-} & \text {on } \partial \Omega, \\
\left.\mathcal{D}_{\Omega}[f]\right|_{ \pm}=\left(\mp \frac{1}{2} I+\mathcal{K}_{\Omega}\right)[f] & \text { on } \partial \Omega,
\end{array}
$$

where $\mathcal{K}_{\Omega}: L^{2}(\partial \Omega) \rightarrow L^{2}(\partial \Omega)$ is defined by

$$
\forall z \in \partial \Omega, \quad \mathcal{K}_{\Omega}[f](z)=\int_{\partial \Omega} \frac{\partial}{\partial \nu(y)} \Gamma_{z}(y) f(y) d s(y) .
$$


Lemma 3.2. Let $d=2,3$. The single layer potential verifies, for all $f \in L^{2}(\partial \Omega)$,

$$
\begin{array}{cc}
\left(-\Delta+k^{2}\right) \mathcal{S}_{\Omega}[f]=0 & \text { in } \mathbb{R}^{d} \backslash \partial \Omega, \\
\left.\mathcal{S}_{\Omega}[f]\right|_{+}=\left.\mathcal{S}_{\Omega}[f]\right|_{-} & \text {on } \partial \Omega .
\end{array}
$$

The single layer potential is therefore well defined on $\partial \Omega$, and hence on $\mathbb{R}^{d}$. Moreover,

$$
\left.\frac{\partial}{\partial \nu} \mathcal{S}_{\Omega}[f]\right|_{ \pm}=\left( \pm \frac{1}{2} I+\mathcal{K}_{\Omega}^{*}\right)[f] \quad \text { on } \partial \Omega,
$$

where $\mathcal{K}_{\Omega}^{*}: L^{2}(\partial \Omega) \rightarrow L^{2}(\partial \Omega)$ is the $L^{2}$-adjoint of the operator $\mathcal{K}_{\Omega}$, i.e.,

$$
\forall z \in \partial \Omega, \quad \mathcal{K}_{\Omega}^{*}[f](z)=\int_{\partial \Omega} \frac{\partial}{\partial \nu(z)} \Gamma_{z}(y) f(y) d s(y) .
$$

Let $G$ be the Green function of problem (3.1), that is, for all $z \in \Omega$, the unique solution to

$$
\begin{cases}-\Delta_{y} G_{z}(y)+k^{2} G_{z}(y)=\delta_{z} & \text { in } \Omega, \\ \ell \frac{\partial G_{z}}{\partial \nu}(y)+G_{z}(y)=0 & \text { on } \partial \Omega .\end{cases}
$$

Lemma 3.3. The operator of kernel $G_{z}(y)$ is the solution operator for problem (3.1):

$$
\forall z \in \Omega, \quad \Phi_{\mathrm{exc}}^{g}(z)=\frac{1}{\ell} \int_{\partial \Omega} G_{z}(y) g(y) d s(y) .
$$

Proof. Since $G_{z}$ and $\Phi_{\mathrm{exc}}^{g}$ are respectively the solutions to problems (3.3) and (3.1), we have the equation

$$
\Phi_{\mathrm{exc}}^{g}(z)=\int_{\Omega}\left[\left(-\Delta_{y} G_{z}(y)+k^{2} G_{z}(y)\right) \Phi_{\mathrm{exc}}^{g}(y)-\left(-\Delta \Phi_{\mathrm{exc}}^{g}(y)+k^{2} \Phi_{\mathrm{exc}}^{g}(y)\right) G_{z}(y)\right] d y .
$$

Besides, we can apply Green's formula:

$$
\begin{aligned}
\Phi_{\mathrm{exc}}^{g}(z)=\int_{\Omega} & {\left[-\Delta_{y} G_{z}(y) \Phi_{\mathrm{exc}}^{g}(y)+\Delta \Phi_{\mathrm{exc}}^{g}(y) G_{z}(y)\right] d y } \\
= & \int_{\partial \Omega}\left[-\frac{\partial G_{z}(y)}{\partial \nu} \Phi_{\operatorname{exc}}^{g}(y)+\frac{\partial \Phi_{\mathrm{exc}}^{g}(y)}{\partial \nu} G_{z}(y)\right] d s(y) .
\end{aligned}
$$

Using the boundary conditions that $G_{z}$ and $\Phi_{\text {exc }}^{g}$ verify, we then obtain that

$$
\Phi_{\mathrm{exc}}^{g}(z)=\frac{1}{\ell} \int_{\partial \Omega} G_{z}(y) g(y) d s(y) .
$$

Thanks to the previous lemma, if we know $G_{z}$, we can calculate the excitation light fluence for any source $g$. The following result relates $G_{z}$, the Green function of our problem, to $\Gamma_{z}$, for which we have an explicit formula. It generalizes [7, Lemma 2.15] to the Green function $G_{z}$. 
Proposition 3.4. For $z \in \Omega$ and $y \in \partial \Omega$,

$$
\left(-\frac{I}{2}+\mathcal{K}_{\Omega}+\frac{1}{\ell} \mathcal{S}_{\Omega}\right)\left[G_{z}\right](y)=\Gamma_{z}(y)
$$

More precisely, for any simply connected smooth domain $D$ compactly contained in $\Omega$ and for any $h \in L^{2}(\partial D)$, we have for any $y \in \partial \Omega$ :

$$
\int_{\partial D}\left(-\frac{I}{2}+\mathcal{K}_{\Omega}+\frac{1}{\ell} \mathcal{S}_{\Omega}\right)\left[G_{z}\right](y) h(z) d s(z)=\int_{\partial D} \Gamma_{z}(y) h(z) d s(z) .
$$

Proof. Let $f \in L_{0}^{2}(\partial \Omega)$, where $L_{0}^{2}(\partial \Omega)$ is the set of $L^{2}$ functions in $\Omega$ of mean zero. For $z \in \Omega$ and $y \in \partial \Omega$, we define

$$
u(z):=\int_{\partial \Omega}\left(-\frac{I}{2}+\mathcal{K}_{\Omega}+\frac{1}{\ell} \mathcal{S}_{\Omega}\right)\left[G_{z}\right](y) f(y) d s(y) .
$$

By introducing the adjoint operator, we obtain

$$
u(z)=\int_{\partial \Omega} G_{z}(y)\left(-\frac{I}{2}+\mathcal{K}_{\Omega}^{*}+\frac{1}{\ell} \mathcal{S}_{\Omega}\right)[f](y) d s(y) .
$$

By Lemma 3.3. $u$ is then a solution to the problem

$$
\begin{cases}-\Delta u(y)+k^{2} u(y)=0 & \text { in } \Omega, \\ \frac{\partial u}{\partial \nu}(y)+\frac{1}{\ell} u(y)=\left(-\frac{I}{2}+\mathcal{K}_{\Omega}^{*}+\frac{1}{\ell} \mathcal{S}_{\Omega}\right)[f](y) & \text { on } \partial \Omega .\end{cases}
$$

We know that $\mathcal{S}_{\Omega}[f]$ is a solution to the problem (3.6), thanks to Lemma 3.2. The equation $\left(-\Delta+k^{2}\right) p=0$ in $\Omega$ with the Robin boundary condition, $\partial p / \partial \nu+l p=0$, admits a unique solution, provided that $l>0$. Therefore, we have

$$
\forall z \in \Omega, \quad u(z)=\mathcal{S}_{\Omega}[f](z) .
$$

Since $f$ is arbitrary, we have therefore proved the first part of our proposition.

Let $h \in L^{2}(\partial D)$. By multiplying the last equality by $h$ and integrating on $\partial D$, we obtain

$$
\begin{aligned}
\int_{\partial \Omega} \int_{\partial D}\left(-\frac{I}{2}+\mathcal{K}_{\Omega}+\frac{1}{\ell} \mathcal{S}_{\Omega}\right)\left[G_{z}\right](y) h(z) f(y) d s(z) & d s(y) \\
& =\int_{\partial \Omega} \int_{\partial D} \Gamma_{z}(y) h(z) f(y) d s(z) d s(y),
\end{aligned}
$$

which completes the proof.

According to the previous proposition, the knowledge of $G_{z}$ and therefore of $\Phi_{\mathrm{exc}}^{g}$ requires the inversion of the operator:

$$
-\frac{I}{2}+\mathcal{K}_{\Omega}+\frac{1}{\ell} \mathcal{S}_{\Omega}: L^{2}(\partial \Omega) \rightarrow L^{2}(\partial \Omega)
$$

In the case of circular domains, we can exhibit an explicit formula of the inverse operator. 
Explicit calculation of $G_{z}$ for a circular domain: We assume that the dimension is two and $\Omega$ is the unit disk. In terms of polar coordinates, the fundamental solution $\Gamma_{z}$ to $-\Delta+k^{2}$ has the expression

$$
\forall y(r, \theta) \in \bar{\Omega}, \forall z(R, \phi) \in \bar{\Omega}, \quad \Gamma_{z}(y)=\frac{i}{4} H_{0}^{(1)}\left(i k\left|r e^{i \theta}-R e^{i \phi}\right|\right) .
$$

Graf's formula [1, Formula (9.1.79)] gives us the following decomposition of $\Gamma_{z}$ :

$$
H_{0}^{(1)}\left(i k\left|r e^{i \theta}-R e^{i \phi}\right|\right)=\sum_{m \in \mathbb{Z}} H_{m}^{(1)}(i k r) J_{m}(i k R) e^{i m(\theta-\phi)}, \quad r>R,
$$

with $H_{m}^{(1)}$ and $J_{m}$ being respectively the Hankel and Bessel functions of the first kind of order $m$.

For all $g \in L^{2}(] 0,2 \pi[)$, we introduce the Fourier coefficients:

$$
\forall m \in \mathbb{Z}, \quad \hat{g}(m)=\frac{1}{2 \pi} \int_{0}^{2 \pi} g(\phi) e^{-i m \phi} d \phi
$$

and have then

$$
g(\phi)=\sum_{m=-\infty}^{\infty} \hat{g}(m) e^{i m \phi} \text { in } L^{2} .
$$

Let $D$ be the disk with radius $R$ and center 0 . For $y(r, \theta) \in \bar{\Omega}$,

$$
\begin{aligned}
\mathcal{S}_{D}[g](y) & =\frac{i R}{4} \int_{0}^{2 \pi} H_{0}^{(1)}\left(i k\left|r e^{i \theta}-R e^{i \phi}\right|\right) g(\phi) d \phi, \\
& =\frac{i R}{4} \sum_{m=-\infty}^{\infty} H_{m}^{(1)}(i k r) J_{m}(i k R) e^{i m \theta} \int_{0}^{2 \pi} g(\phi) e^{-i m \phi} d \phi \\
& =\frac{i R \pi}{2} \sum_{m=-\infty}^{\infty} H_{m}^{(1)}(i k r) J_{m}(i k R) \hat{g}(m) e^{i m \theta} .
\end{aligned}
$$

For $y(1, \theta) \in \partial \Omega$, we therefore obtain

$$
\mathcal{S}_{D}[g](y)=\sum_{m=-\infty}^{\infty} \widehat{\mathcal{S}_{D}}(m) \hat{g}(m) e^{i m \theta},
$$

with

and, analogously,

$$
\forall m \in \mathbb{Z}, \quad \widehat{\mathcal{S}_{D}}(m)=\frac{i R \pi}{2} H_{m}^{(1)}(i k) J_{m}(i k R)
$$

$$
\mathcal{S}_{\Omega}[g](y)=\sum_{m=-\infty}^{\infty} \widehat{\mathcal{S}_{\Omega}}(m) \hat{g}(m) e^{i m \theta}
$$

with

$$
\forall m \in \mathbb{Z}, \quad \widehat{\mathcal{S}_{\Omega}}(m)=\frac{i \pi}{2} H_{m}^{(1)}(i k) J_{m}(i k) .
$$

We can prove in a similar way that

$$
\mathcal{K}_{\Omega}[g](y)=\sum_{m=-\infty}^{\infty} \widehat{\mathcal{K}_{\Omega}}(m) \hat{g}(m) e^{i m \theta},
$$


with

$$
\forall m \in \mathbb{Z}, \quad \widehat{\mathcal{K}_{\Omega}}(m)=\frac{-k \pi}{2} H_{m}^{(1)}(i k) J_{m}^{\prime}(i k) .
$$

Using Proposition 3.4, we can express the Fourier coefficients of the operator with kernel $G_{z}(y)$ for all $z(R, \theta) \in \partial D$, defined by

$$
\int_{\partial \Omega} G_{z}(y) g(y) d s(y)=\sum_{m=-\infty}^{\infty} \widehat{G}(m) \hat{g}(m) e^{i m \theta},
$$

as follows:

$$
\forall m \in \mathbb{Z}, \quad \widehat{G}(m)=\frac{\widehat{\mathcal{S}_{D}}(m)}{\widehat{\mathcal{K}_{\Omega}}(m)+\frac{1}{\ell} \widehat{\mathcal{S}_{\Omega}}(m)}
$$

That is,

$$
\forall m \in \mathbb{Z}, \quad \widehat{G}(m)=\frac{J_{m}(i k R)}{i k J_{m}^{\prime}(i k)+\frac{1}{\ell} J_{m}(i k)} .
$$

Moreover, the function $\Phi_{\text {exc }}^{g}$ defined by (3.4) can be written as

$$
\Phi_{\mathrm{exc}}^{g}(R, \theta)=\sum_{m=-\infty}^{\infty} \frac{J_{m}(i k R)}{i k \ell J_{m}^{\prime}(i k)+J_{m}(i k)} \hat{g}(m) e^{i m \theta} .
$$

When $\Omega$ is approximated by the unit disk, we have shown that we can easily invert our operator (3.7) and obtain an explicit formula of our Green's function $G_{z}$. We can then calculate the excitation light fluence for any source $g$ in this particular case. The same result holds for the unit sphere; see Appendix A.

3.2. Expression of $c_{\mathrm{ffr}}$. Recall that the concentration of fluorophores $c_{\mathrm{fr}}$ can be expressed as

$$
c_{\mathrm{flr}}=\left.\delta[u]\right|_{\partial C},
$$

where $\delta$ is a constant and $u$, the voltage potential in our domain, satisfies (2.4).

Let $L_{0}^{2}(\partial C):=\left\{\Psi \in L^{2}(\partial C): \int_{\partial C} \Psi=0\right\}$. Let $\Gamma^{(0)}$ be the fundamental solution to $\Delta$ in $\mathbb{R}^{d}$ :

$$
\Gamma^{(0)}(x):= \begin{cases}\frac{1}{2 \pi} \log |x|, & d=2, \\ -\frac{1}{4 \pi|x|}, & d=3 .\end{cases}
$$

Analogously to Section 3 , we introduce the layer potentials $\mathcal{S}_{C}^{(0)}, \mathcal{S}_{\Omega}^{(0)}, \mathcal{D}_{C}^{(0)}, \mathcal{D}_{\Omega}^{(0)}, \mathcal{K}_{C}^{(0)}$, and $\left(\mathcal{K}_{C}^{(0)}\right)^{*}$ associated with $\Gamma^{(0)}$. The following proposition from [21] gives us a representation formula for the voltage potential in $\Omega$.

Proposition 3.5. There exists at most one solution $u$ to the problem (2.4) and it satisfies the following representation formula:

$$
\forall x \in \Omega, \quad u(x)=H(x)+\mathcal{D}_{C}^{(0)}[\Psi](x),
$$

where the harmonic function $H$ is given by

$$
\forall x \in \mathbb{R}^{2} \backslash \partial \Omega, \quad H(x)=-\mathcal{S}_{\Omega}^{(0)}\left[g_{\text {ele }}\right](x)+\mathcal{D}_{\Omega}^{(0)}\left[\left.u\right|_{\partial \Omega}\right](x),
$$


and $\Psi \in L_{0}^{2}(\partial C)$ satisfies the integral equation

$$
\Psi+\beta \frac{\partial \mathcal{D}_{C}^{(0)}[\Psi]}{\partial \nu}=-\beta \frac{\partial H}{\partial \nu} \quad \text { on } \partial C .
$$

The decomposition in (3.10) is unique. Furthermore, the following identity holds:

$$
\forall x \in \mathbb{R}^{2} \backslash \bar{\Omega}, \quad u(x)=H(x)+\mathcal{D}_{C}^{(0)}[\Psi](x)=0 .
$$

Since the normal derivative of the layer potential is continuous across its boundary, the representation formula (3.10) gives us an expression for $\left.\frac{\partial u}{\partial \nu}\right|_{\partial C}$ and hence for $c_{\mathrm{flr}}$ thanks to (2.4) and (2.5). For a given applied electric field $g_{\text {ele }}$ and cell $C$, one can therefore compute the fluorophore concentration $c_{\mathrm{flr}}$ on $\partial C$.

3.3. Expression of $\Phi_{\mathrm{emt}}^{g}$. The emitted light fluence $\Phi_{\mathrm{emt}}^{g}$ due to an excitation $g$ is the solution to the following problem:

$$
\begin{cases}-\Delta \Phi_{\mathrm{emt}}^{g}(y)+k^{2} \Phi_{\mathrm{emt}}^{g}(y)=\frac{\tilde{\gamma}}{D} c_{\mathrm{flr}}(y) \Phi_{\mathrm{exc}}^{g}(y) & \text { in } \Omega \\ \ell \frac{\partial \Phi_{\mathrm{emt}}^{g}}{\partial \nu}(y)+\Phi_{\mathrm{emt}}^{g}(y)=0 & \text { on } \partial \Omega\end{cases}
$$

where $\Phi_{\text {exc }}^{g}$ is the excitation light fluence launched by the source $g$ in $\Omega$.

The measured quantity on $\partial \Omega$ is

$$
I_{\mathrm{emt}}^{g}=-\left.D \frac{\partial \Phi_{\mathrm{emt}}^{g}}{\partial \nu}\right|_{\partial \Omega},
$$

which is the outgoing light intensity determined from Fick's law. It is worth mentioning that, in our coupled diffusion equations model, if $\ell \neq 0$, then knowing $\Phi_{\mathrm{emt}}^{g}$ or $\partial \Phi_{\mathrm{emt}}^{g} / \partial \nu$ on $\partial \Omega$ is mathematically the same.

Proposition 3.6. The emitted light fluence $\Phi_{\mathrm{emt}}^{g}$ can be expressed as a function of $G_{z}$ and $\Phi_{\mathrm{exc}}^{g}$ as follows:

$$
\forall z \in \bar{\Omega}, \quad \Phi_{\mathrm{emt}}^{g}(z)=\int_{\partial C} \frac{\tilde{\gamma}}{D} G_{z}(y) c_{\mathrm{flr}}(y) \Phi_{\mathrm{exc}}^{g}(y) d s(y),
$$

where $\partial C$ is the cell membrane.

Proof. Since $G$ and $\Phi_{\mathrm{emt}}^{g}$ are the solutions to the problems (3.3) and (3.13), we have

$$
\begin{gathered}
\Phi_{\mathrm{emt}}^{g}(z)-\int_{\Omega} \frac{\tilde{\gamma}}{D} G_{z}(y) c_{\mathrm{frr}}(y) \Phi_{\mathrm{exc}}^{g}(y) d y=\int_{\Omega}\left[\left(-\Delta_{y} G_{z}(y)+k^{2} G_{z}(y)\right) \Phi_{\mathrm{emt}}^{g}(y)\right. \\
\left.-G_{z}(y)\left(-\Delta \Phi_{\mathrm{emt}}^{g}(y)+k^{2} \Phi_{\mathrm{emt}}^{g}(y)\right)\right] d y .
\end{gathered}
$$

Besides, we can apply Green's formula:

$$
\begin{aligned}
\Phi_{\mathrm{emt}}^{g}(z)-\int_{\Omega} \frac{\tilde{\gamma}}{D} G_{z}(y) c_{\mathrm{flr}}(y) \Phi_{\mathrm{exc}}^{g}(y) d y \\
\quad=\int_{\partial \Omega}\left[-\frac{\partial G_{z}(y)}{\partial \nu} \Phi_{\mathrm{emt}}^{g}(y)+G_{z}(y) \frac{\partial \Phi_{\mathrm{emt}}^{g}(y)}{\partial \nu}\right] d s(y) .
\end{aligned}
$$


Using the boundary conditions that $G_{z}$ and $\Phi_{\mathrm{emt}}^{g}$ verify, we then obtain

$$
\begin{aligned}
\Phi_{\mathrm{emt}}^{g}(z)-\int_{\Omega} \frac{\tilde{\gamma}}{D} G_{z}(y) c_{\mathrm{flr}}(y) \Phi_{\mathrm{exc}}^{g}(y) d y & =\int_{\partial \Omega}\left[\frac{1}{\ell} G_{z}(y) \Phi_{\mathrm{emt}}^{g}(y)+G_{z}(y) \frac{\partial \Phi_{\mathrm{emt}}^{g}(y)}{\partial \nu}\right] d s(y) \\
& =0 .
\end{aligned}
$$

Since the concentration of the fluorophores is zero except on $\partial C$, we get finally the formula

$$
\forall z \in \bar{\Omega}, \quad \Phi_{\mathrm{emt}}^{g}(z)=\int_{\partial C} \frac{\tilde{\gamma}}{D} G_{z}(y) c_{\mathrm{ffr}}(y) \Phi_{\mathrm{exc}}^{g}(y) d s(y)
$$

By combining the results of the first section and of this last section for a given concentration of fluorophore $c_{\mathrm{fr}}$ and an excitation $g$, we can express $\Phi_{\mathrm{emt}}^{g}$ at any point of $\bar{\Omega}$, and in particular on $\partial \Omega$. Moreover, section 3.2 gives us a unique formula for the fluorophore concentration for given $g_{\text {ele }}$ and $C$. If we couple these two formulas, we solve our forward problem.

4. Inverse problem. The shape and position of the cell $C$ are now considered to be unknown. We illuminate our domain with a light source $g$ and apply an electric field $g_{\text {ele }}$ at its boundary. We measure an outgoing light intensity $I_{\mathrm{emt}}^{g}$. Our goal is to reconstruct the concentration of fluorophore $c_{\mathrm{fr}}$. We will thus have an image of the membrane potential changes and hence locate the cell. In this section we consider only the two-dimensional case. We start with the reconstruction of the cell membrane $\partial C$ in the case when it is assumed to be a perturbation of a disk. We derive analytical formulas for the resolving power of the proposed imaging method in two different regimes. Then we extend our results to arbitrary shapes. In three dimensions, similar results hold and analytical formulas for the resolving power of the imaging method can be derived for $\partial C$ being a perturbation of a sphere.

4.1. Problem formulation. The excitation light fluence, $\Phi_{\mathrm{exc}}^{f}$, due to a source $f \in$ $L^{2}(\partial \Omega)$, is the solution to

$$
\begin{cases}-\Delta \Phi_{\mathrm{exc}}^{f}(y)+k^{2} \Phi_{\mathrm{exc}}^{f}(y)=0 & \text { in } \Omega, \\ \ell \frac{\partial \Phi_{\mathrm{exc}}^{f}}{\partial \nu}(y)+\Phi_{\mathrm{exc}}^{f}(y)=f & \text { on } \partial \Omega .\end{cases}
$$

We denote by $\Phi_{\operatorname{exc}}^{g}$ the excitation light fluence due to an excitation $g \in L^{2}(\partial \Omega)$. The emitted light fluence, $\Phi_{\mathrm{emt}}^{g}$, due to the excitation of the fluorophores by $\Phi_{\mathrm{exc}}^{g}$, verifies

$$
\begin{cases}-\Delta \Phi_{\mathrm{emt}}^{g}(y)+k^{2} \Phi_{\mathrm{emt}}^{g}(y)=\frac{\tilde{\gamma}}{D} c_{\mathrm{flr}}(y) \Phi_{\mathrm{exc}}^{g}(y) & \text { in } \Omega \\ \ell \frac{\partial \Phi_{\mathrm{emt}}^{g}}{\partial \nu}(y)+\Phi_{\mathrm{emt}}^{g}(y)=0 & \text { on } \partial \Omega .\end{cases}
$$

By multiplying the last equation by $\Phi_{\mathrm{exc}}^{f}$ and integrating on our domain $\Omega$, we obtain the following formula:

$$
\int_{\Omega} \frac{\tilde{\gamma}}{D} c_{\mathrm{flr}}(y) \Phi_{\mathrm{exc}}^{g}(y) \Phi_{\mathrm{exc}}^{f}(y) d y=\int_{\Omega}\left[-\Delta \Phi_{\mathrm{emt}}^{g}(y) \Phi_{\mathrm{exc}}^{f}(y)+k^{2} \Phi_{\mathrm{emt}}^{g}(y) \Phi_{\mathrm{exc}}^{f}(y)\right] d y .
$$


From the first equation, we know that in $\Omega$,

$$
k^{2} \Phi_{\mathrm{exc}}^{f} \Phi_{\mathrm{emt}}^{g}=\Delta \Phi_{\mathrm{exc}}^{f} \Phi_{\mathrm{emt}}^{g} .
$$

Hence, we have

$$
\int_{\Omega} \frac{\tilde{\gamma}}{D} c_{\mathrm{flr}}(y) \Phi_{\mathrm{exc}}^{g}(y) \Phi_{\mathrm{exc}}^{f}(y) d y=\int_{\Omega}\left[-\Delta \Phi_{\mathrm{emt}}^{g}(y) \Phi_{\mathrm{exc}}^{f}(y)+\Delta \Phi_{\mathrm{exc}}^{f}(y) \Phi_{\mathrm{emt}}^{g}(y)\right] d y .
$$

Green's formula gives us

$$
\int_{\Omega} \frac{\tilde{\gamma}}{D} c_{\mathrm{flr}}(y) \Phi_{\mathrm{exc}}^{g}(y) \Phi_{\mathrm{exc}}^{f}(y) d y=\int_{\partial \Omega}\left[-\frac{\partial \Phi_{\mathrm{emt}}^{g}}{\partial \nu}(y) \Phi_{\mathrm{exc}}^{f}(y)+\frac{\partial \Phi_{\mathrm{exc}}^{f}}{\partial \nu}(y) \Phi_{\mathrm{emt}}^{g}(y)\right] d s(y) .
$$

We use the boundary conditions of our two equations and obtain that

$$
\int_{\Omega} \frac{\tilde{\gamma}}{D} c_{\mathrm{flr}}(y) \Phi_{\mathrm{exc}}^{g}(y) \Phi_{\mathrm{exc}}^{f}(y) d y=\frac{1}{\ell} \int_{\partial \Omega} f(y) \Phi_{\mathrm{emt}}^{g}(y) d s(y) .
$$

The concentration of the fluorophores is zero except on $\partial C$, so we get finally the following proposition.

Proposition 4.1. Let $f$ and $g$ be in $L^{2}(\partial \Omega)$. The outgoing light intensity $I_{\mathrm{emt}}^{g}=$ $-D \frac{\partial \Phi_{\text {emt }}^{g}}{\partial \nu}$ measured on $\partial \Omega$ satisfies the formula

$$
\int_{\partial C} \tilde{\gamma} c_{\mathrm{frr}}(y) \Phi_{\mathrm{exc}}^{g}(y) \Phi_{\mathrm{exc}}^{f}(y) d s(y)=\int_{\partial \Omega} f(y) I_{\mathrm{emt}}^{g}(y) d s(y) .
$$

This formula also holds for $\ell=0$.

For two chosen excitations $f, g \in L^{2}(\partial \Omega)$ and a measured outgoing light intensity $I_{\mathrm{emt}}^{g}$, we can compute the integral $\int_{\partial \Omega} f(y) I_{\mathrm{emt}}^{g}(y) d s(y)$, and hence, thanks to the last formula, $\int_{\partial C} \tilde{\gamma} c_{\mathrm{flr}}(y) \Phi_{\mathrm{exc}}^{g}(y) \Phi_{\mathrm{exc}}^{f}(y) d s(y)$. Recall that the constant $\tilde{\gamma}$ is assumed to be known. Then, if we properly choose $f$ and $g$, we will be able to reconstruct $c_{\mathrm{frr}} \nVdash_{\partial C}$ and therefore to image the cell membrane $\partial C$.

4.2. Reconstruction of the cell membrane: case of a perturbed disk. We consider a circular cell $C$ with radius $R$. We choose to excite our medium with a source given by

$$
f_{n}(\phi)=E_{n} e^{i n \phi},
$$

for $n \in \mathbb{Z}, \phi \in[0,2 \pi]$ and $E_{n}:=i k \ell J_{n}^{\prime}(i k)+J_{n}(i k)$. It gives us, thanks to formula (3.8), the excitation light fluence $\Phi_{\mathrm{exc}}^{n}$ :

$$
\forall \theta \in[0,2 \pi], \quad \Phi_{\mathrm{exc}}^{n}(R, \theta)=J_{n}(i k R) e^{-i n \theta} .
$$

Let $\Phi_{\mathrm{emt}}^{n}$ be the emitted light fluence, and let $I_{\mathrm{emt}}^{n}=-\left.D \frac{\partial \Phi_{\mathrm{emt}}^{n}}{\partial \nu}\right|_{\partial \Omega}$ be the outgoing light intensity measured at $\partial \Omega$ when the cell occupies $C$ and the source $f_{n}$ is applied at $\partial \Omega$. It follows from (4.1) that

$$
\int_{\partial C} \tilde{\gamma} c_{\mathrm{frr}}(\theta) \Phi_{\mathrm{exc}}^{n}(R, \theta) \Phi_{\mathrm{exc}}^{m}(R, \theta) R d \theta=2 \pi E_{m} \widehat{I_{\mathrm{emt}}^{n}}(m) .
$$


Besides, we also have

$$
\int_{\partial C} \tilde{\gamma} c_{\mathrm{flr}}(\theta) \Phi_{\mathrm{exc}}^{n}(R, \theta) \Phi_{\mathrm{exc}}^{m}(R, \theta) R d \theta=2 \pi \tilde{\gamma} R J_{n}(i k R) J_{m}(i k R) \widehat{c_{\mathrm{flr}}}(n+m) .
$$

Let $C_{\epsilon}$ be an $\epsilon$-perturbation of $C$; i.e., there is $h \in \mathcal{C}^{2}([0,2 \pi])$ such that $\partial C_{\epsilon}$ is given by

$$
\partial C_{\epsilon}=\left\{\tilde{x} ; \tilde{x}(\theta)=(R+\epsilon h(\theta)) e_{r}, \theta \in[0,2 \pi]\right\},
$$

with $\left(e_{r}, e_{\theta}\right)$ being the basis of polar coordinates.

Our goal is to reconstruct the shape deformation $h$ of our cell. Let $\Phi_{\mathrm{emt}, \epsilon}^{n}$ be the emitted light fluence, and let $I_{\mathrm{emt}, \epsilon}^{n}=-\left.D \frac{\partial \Phi_{\mathrm{emt}, \epsilon}^{n}}{\partial \nu}\right|_{\partial \Omega}$ be the outgoing light intensity measured at the boundary of our domain $\Omega$ when the cell occupies $C_{\epsilon}$ and the source $f_{n}$ is applied at $\partial \Omega$. Again, it follows from (4.1) that

$$
\int_{\partial C_{\epsilon}} \tilde{\gamma} \widetilde{c_{\mathrm{flr}}}(x) \Phi_{\mathrm{exc}}^{n}(x) \Phi_{\mathrm{exc}}^{m}(x) d s(x)=2 \pi E_{m} \widehat{I_{\mathrm{emt}, \epsilon}^{n}}(m) .
$$

On the other hand, we have

$$
\int_{\partial C_{\epsilon}} \tilde{\gamma} \widetilde{c_{\mathrm{fr}}}(x) \Phi_{\mathrm{exc}}^{n}(x) \Phi_{\mathrm{exc}}^{m}(x) d s(x)=\int_{\partial C} \tilde{\gamma} \widetilde{c_{\mathrm{fr}}}(\tilde{x}) J_{n}(i k \tilde{R}(\theta)) J_{m}(i k \tilde{R}(\theta)) e^{-i(n+m) \theta} d s_{\epsilon}(\tilde{x}),
$$

where $\tilde{R}(\theta)=R+\epsilon h(\theta)$ and $\widetilde{c_{\mathrm{flr}}}$ is the concentration of fluorophores on the deformed cell membrane $\partial C_{\epsilon}$.

We want to compute the first-order approximation of our integral (4.4). TaylorLagrange's theorem gives us the following expansions for all $N \in \mathbb{N}$ :

$$
\begin{aligned}
& J_{m}(i k \tilde{R})=\sum_{p=0}^{N} \frac{(i k \epsilon h(\theta))^{p}}{p !} J_{m}^{(p)}(i k R)+o\left(\epsilon^{N}\right), \\
& J_{n}(i k \tilde{R})=\sum_{p=0}^{N} \frac{(i k \epsilon h(\theta))^{p}}{p !} J_{n}^{(p)}(i k R)+o\left(\epsilon^{N}\right) .
\end{aligned}
$$

In particular, at first order,

$$
\begin{aligned}
& J_{m}(i k \tilde{R})=J_{m}(i k R)+\epsilon i k h(\theta) J_{m}^{\prime}(i k R)+o(\epsilon), \\
& J_{n}(i k \tilde{R})=J_{n}(i k R)+\epsilon i k h(\theta) J_{n}^{\prime}(i k R)+o(\epsilon) .
\end{aligned}
$$

We can easily get an expansion for the length element $d s_{\epsilon}(\tilde{y})$ for $\tilde{y} \in \partial C_{\epsilon}$ :

$$
d s_{\epsilon}(\tilde{y})=\left|\tilde{x^{\prime}}(\theta)\right| d \theta=\left((R+\epsilon h(\theta))^{2}+\left(\epsilon h^{\prime}(\theta)\right)^{2}\right)^{\frac{1}{2}} d \theta=\sum_{n=0}^{\infty} \epsilon^{n} \sigma^{(n)}(\theta) d \theta
$$

where $\sigma^{(n)}$ are functions bounded independently of $n$ and, at first order, we have

$$
d s_{\epsilon}(\tilde{y})=R d \theta+\epsilon h(\theta) d \theta+o(\epsilon) .
$$


4.2.1. High-order terms in the expansion of $\widetilde{c_{\mathrm{flr}}}$. We denote $u_{\epsilon}$ (resp. $u$ ) the voltage potential in our medium when the cell occupies $C_{\epsilon}$ (resp. $C$ ). We assume, thanks to (2.5), that our concentration of fluorophores $\widetilde{c_{f f r}}\left(\right.$ resp. $\left.c_{\mathrm{frr}}\right)$ on $\partial C_{\epsilon}$ (resp. $\partial C$ ) is given by

$$
\begin{aligned}
& \widetilde{c_{\mathrm{fl} r}} & =\left.\delta\left[u_{\epsilon}\right]\right|_{\partial C_{\epsilon}} \\
\text { resp. } & c_{\mathrm{flr}} & =\left.\delta[u]\right|_{\partial C} .
\end{aligned}
$$

To find the first-order term in the expansion of $\widetilde{c_{\mathrm{fl}}}$, we must therefore expand at first $u_{\epsilon}$. Similar problems have been considered in [10,11]. Nevertheless, our derivations, based on a layer potential technique, differ significantly from those in [10,11.

We know from Proposition 3.5 that $u_{\epsilon}$ (resp. $u$ ) admits the following representation formula:

$$
\begin{aligned}
& \forall x \in \Omega, u_{\epsilon}(x)=H_{\epsilon}(x)+\mathcal{D}_{C_{\epsilon}}^{(0)}\left[\Psi_{\epsilon}\right](x) \\
& \text { resp. } \forall x \in \Omega, \quad u(x)=H(x)+\mathcal{D}_{C}^{(0)}[\Psi](x),
\end{aligned}
$$

where the harmonic function $H_{\epsilon}$ (resp. $H$ ) is given by

$$
\begin{aligned}
\forall x & \in \mathbb{R}^{2} \backslash \partial \Omega, \quad H_{\epsilon}(x)=-\mathcal{S}_{\Omega}^{(0)}\left[g_{\text {ele }}\right](x)+\mathcal{D}_{\Omega}^{(0)}\left[\left.u_{\epsilon}\right|_{\partial \Omega}\right](x) \\
\text { resp. } \quad \forall x & \in \mathbb{R}^{2} \backslash \partial \Omega, \quad H(x)=-\mathcal{S}_{\Omega}^{(0)}\left[g_{\text {ele }}\right](x)+\mathcal{D}_{\Omega}^{(0)}\left[\left.u\right|_{\partial \Omega}\right](x),
\end{aligned}
$$

and $\Psi_{\epsilon} \in L_{0}^{2}\left(\partial C_{\epsilon}\right)$ (resp. $\Psi \in L_{0}^{2}(\partial C)$ ) satisfies the integral equation

$$
\begin{gathered}
\Psi_{\epsilon}+\beta \frac{\partial \mathcal{D}_{C_{\epsilon}}^{(0)}\left[\Psi_{\epsilon}\right]}{\partial \tilde{\nu}}=-\beta \frac{\partial H_{\epsilon}}{\partial \tilde{\nu}} \quad \text { on } \partial C_{\epsilon} \\
\text { resp. } \Psi+\beta \frac{\partial \mathcal{D}_{C}^{(0)}[\Psi]}{\partial \nu}=-\beta \frac{\partial H}{\partial \nu} \quad \text { on } \partial C,
\end{gathered}
$$

where $\tilde{\nu}(\tilde{x})$ (resp. $\nu(x))$ denotes the outward unit normal to $\partial C_{\epsilon}($ resp. $\partial C$ ) at $\tilde{x}$ (resp. $x)$.

Therefore we obtain, for all $x \in \Omega$,

$$
u_{\epsilon}(x)-u(x)=\mathcal{D}_{\Omega}^{(0)}\left[\left.u_{\epsilon}\right|_{\partial \Omega}-\left.u\right|_{\partial \Omega}\right](x)+\mathcal{D}_{C_{\epsilon}}^{(0)}\left[\Psi_{\epsilon}\right](x)-\mathcal{D}_{C}^{(0)}[\Psi](x),
$$

and, on $\partial \Omega$,

$$
u_{\epsilon}(x)-u(x)=\left(\frac{I}{2}+\mathcal{K}_{\Omega}^{(0)}\right)\left[u_{\epsilon}-u\right](x)+\mathcal{D}_{C_{\epsilon}}^{(0)}\left[\Psi_{\epsilon}\right](x)-\mathcal{D}_{C}^{(0)}[\Psi](x) .
$$

Our first step is to find high-order terms in the expansion of $\Psi_{\epsilon}$. We define the operator $\mathcal{L}_{\epsilon}(\operatorname{resp} . \mathcal{L})$ on $L^{2}\left(\partial C_{\epsilon}\right)\left(\right.$ resp. $\left.L^{2}(\partial C)\right)$ by

$$
\begin{aligned}
\mathcal{L}_{\epsilon}[f] & =\frac{\partial \mathcal{D}_{C_{\epsilon}}^{(0)}[f]}{\partial \tilde{\nu}} \\
\text { resp. } \quad & \mathcal{L}[f]=\frac{\partial \mathcal{D}_{C}^{(0)}[f]}{\partial \nu} .
\end{aligned}
$$

Proposition 4.2. Let $D$ be a bounded $\mathcal{C}^{2, \eta}$ - domain in $\mathbb{R}^{2}$ for $0<\eta<1$. We denote by $L_{D}$ the normal derivative of the double layer potential on $D, L_{D}:=\partial \mathcal{D}_{D}^{(0)} / \partial \nu$. Then, $I+\beta L_{D}: \mathcal{C}^{2, \eta} \rightarrow \mathcal{C}^{1, \eta}$ is a bounded operator and has a bounded inverse. 
Proof. The boundedness of $L_{D}: \mathcal{C}^{2, \eta} \rightarrow \mathcal{C}^{1, \eta}$ is proved in [12. Note that since $L_{D}$ is not a compact operator, we cannot apply the Fredholm alternative. However, $L_{D}$ is positive [27, and the proposition follows since $\beta>0$.

For $f \in \mathcal{C}^{2, \eta}\left(\partial C_{\epsilon}\right), \tilde{x} \in \partial C_{\epsilon}, \mathcal{L}_{\epsilon}$ has the following expression [21]:

$$
\begin{aligned}
\frac{\partial \mathcal{D}_{C_{\epsilon}}^{(0)}[f]}{\partial \nu}(x)= & -\frac{1}{2 \pi} \int_{\partial D} \frac{\langle\tilde{\nu}(\tilde{x}), \tilde{\nu}(\tilde{y})\rangle}{|\tilde{x}-\tilde{y}|^{2}}(f(\tilde{y})-f(\tilde{x})) d s_{\epsilon}(\tilde{y}) \\
& +\frac{1}{\pi} \int_{\partial D} \frac{\langle\tilde{x}-\tilde{y}, \tilde{\nu}(\tilde{x})\rangle\langle\tilde{x}-\tilde{y}, \tilde{\nu}(\tilde{y})\rangle}{|\tilde{x}-\tilde{y}|^{4}}(f(\tilde{y})-f(\tilde{x})) d s_{\epsilon}(\tilde{y}) .
\end{aligned}
$$

The outward unit normal to $\partial C$ at $x, \nu(x)$, and the tangential vector, $T(x)$, are, in terms of polar coordinates,

$$
\nu(x)=e_{r}(x), \quad T(x)=e_{\theta}(x) .
$$

The outward unit normal to $\partial C_{\epsilon}$ at $\tilde{x}, \tilde{\nu}(\tilde{x})$, is given by

$$
\tilde{\nu}(\tilde{x})=\frac{R_{-\frac{\pi}{2}}\left(\tilde{x^{\prime}}(\theta)\right)}{\left|\tilde{x^{\prime}}(\theta)\right|},
$$

where $R_{-\frac{\pi}{2}}$ stands for rotation by $-\frac{\pi}{2}$. In our case, we then have

$$
\tilde{\nu}(\tilde{x})=\frac{(R+\epsilon h(\theta)) e_{r}-\epsilon h^{\prime}(\theta) e_{\theta}}{\left((R+\epsilon h(\theta))^{2}+\left(\epsilon h^{\prime}(\theta)\right)^{2}\right)^{\frac{1}{2}}} .
$$

We can expand $\tilde{\nu}(\tilde{x})$ for $x \in \partial C$ as follows:

$$
\tilde{\nu}(\tilde{x})=\sum_{n=0}^{\infty} \epsilon^{n} \nu^{(n)}(\theta),
$$

where the vector-valued functions $\nu^{(n)}$ are uniformly bounded independently of $n$.

In particular, at first order, $\tilde{\nu}(\tilde{x})$ for $\tilde{x} \in \partial C_{\epsilon}$ is given by

$$
\tilde{\nu}(\tilde{x})=e_{r}-\frac{h^{\prime}(\theta)}{R} e_{\theta}+o(\epsilon)
$$

Set $\tilde{x}, \tilde{y} \in \partial C_{\epsilon}$. We have

$$
\tilde{x}-\tilde{y}=R\left(e_{r}(x)-e_{r}(y)\right)+\epsilon\left(h\left(\theta^{x}\right) e_{r}(x)-h\left(\theta^{y}\right) e_{r}(y)\right) .
$$

If we denote

$$
c=\cos \left(\theta^{x}-\theta^{y}\right), \quad s=\sin \left(\theta^{x}-\theta^{y}\right),
$$

then we obtain

$$
\begin{aligned}
|\tilde{x}-\tilde{y}|^{2}=2 R^{2}(1-c)+2 \epsilon R(1-c) & \left(h\left(\theta^{x}\right)+h\left(\theta^{y}\right)\right) \\
+ & \epsilon^{2}\left(h\left(\theta^{x}\right)^{2}+h\left(\theta^{y}\right)^{2}-2 h\left(\theta^{x}\right) h\left(\theta^{y}\right) c\right),
\end{aligned}
$$

and

$$
\frac{1}{|\tilde{x}-\tilde{y}|^{2}}=\frac{1}{2 R^{2}(1-c)} \frac{1}{1+\epsilon F\left(\theta^{x}, \theta^{y}\right)+\epsilon^{2} G\left(\theta^{x}, \theta^{y}\right)}
$$

where

$$
F\left(\theta^{x}, \theta^{y}\right)=\frac{\left(h\left(\theta^{x}\right)+h\left(\theta^{y}\right)\right)}{R}, \quad G\left(\theta^{x}, \theta^{y}\right)=\frac{\left\langle h\left(\theta^{x}\right) e_{r}(x)-h\left(\theta^{y}\right) e_{r}(y)\right\rangle^{2}}{2 R^{2}(1-c)} .
$$


Likewise, we write

$$
\frac{1}{|\tilde{x}-\tilde{y}|^{4}}=\frac{1}{4 R^{4}(1-c)^{2}} \frac{1}{\left(1+\epsilon F\left(\theta^{x}, \theta^{y}\right)+\epsilon^{2} G\left(\theta^{x}, \theta^{y}\right)\right)^{2}} .
$$

It follows, from (4.12), (4.7) and (4.17) that

$$
\begin{aligned}
& \frac{\langle\tilde{\nu}(\tilde{x}), \tilde{\nu}(\tilde{y})\rangle}{|\tilde{x}-\tilde{y}|^{2}} d s_{\epsilon}(\tilde{y})=\frac{K_{0}+\epsilon K_{1}+\epsilon^{2} K_{2}}{2 R^{2}(1-c)} \\
& \quad \times \frac{1}{1+\epsilon F\left(\theta^{x}, \theta^{y}\right)+\epsilon^{2} G\left(\theta^{x}, \theta^{y}\right)} \frac{R}{\left(\left(R+\epsilon h\left(\theta^{x}\right)\right)^{2}+\left(\epsilon h^{\prime}\left(\theta^{x}\right)\right)^{2}\right)^{\frac{1}{2}}} R d \theta^{y},
\end{aligned}
$$

where

$$
\begin{aligned}
& K_{0}=c \\
& K_{1}=\frac{1}{R}\left[\left(h\left(\theta^{x}\right)+h\left(\theta^{y}\right)\right) c+\left(h^{\prime}\left(\theta^{x}\right)-h^{\prime}\left(\theta^{y}\right)\right) s\right], \\
& K_{2}=\frac{h^{\prime}\left(\theta^{x}\right) h^{\prime}\left(\theta^{y}\right)}{R^{2}} c .
\end{aligned}
$$

One can see from the previous formulas that the singularity of $\frac{K_{i}}{2 R^{2}(1-c)}$ for $i \in[0,2]$ is of order $O\left(\left|\theta^{x}-\theta^{y}\right|^{-2}\right)$, since $1-c=O\left(\left|\theta^{x}-\theta^{y}\right|^{-2}\right)$.

Likewise, thanks to (4.12), (4.7) and (4.17), we can define $M_{i}$ for $i \in[0,4]$ such that

$$
\begin{aligned}
& \frac{\langle\tilde{x}-\tilde{y}, \tilde{\nu}(\tilde{x})\rangle\langle\tilde{x}-\tilde{y}, \tilde{\nu}(\tilde{y})\rangle}{|\tilde{x}-\tilde{y}|^{4}} d s_{\epsilon}(\tilde{y})=\frac{M_{0}+\epsilon M_{1}+\epsilon^{2} M_{2}+\epsilon^{3} M_{3}+\epsilon^{4} M_{4}}{4 R^{4}(1-c)^{2}} \\
& \quad \times \frac{1}{\left(1+\epsilon F\left(\theta^{x}, \theta^{y}\right)+\epsilon^{2} G\left(\theta^{x}, \theta^{y}\right)\right)^{2}} \frac{R}{\left(\left(R+\epsilon h\left(\theta^{x}\right)\right)^{2}+\left(\epsilon h^{\prime}\left(\theta^{x}\right)\right)^{2}\right)^{\frac{1}{2}}} R d \theta^{y},
\end{aligned}
$$

and the singularity of $\frac{M_{i}}{4 R^{4}(1-c)^{2}}$ for $i \in[0,4]$ is of order $O\left(\left|\theta^{x}-\theta^{y}\right|^{-2}\right)$. Therefore, we get

$$
\begin{aligned}
L_{\epsilon} d s_{\epsilon}(\tilde{y}) & =\frac{N_{0}+\epsilon N_{1}+\epsilon^{2} N_{2}+\epsilon^{3} N_{3}+\epsilon^{4} N_{4}}{2 R^{4}(1-c)^{2}} \\
& \times \frac{1}{\left(1+\epsilon F\left(\theta^{x}, \theta^{y}\right)+\epsilon^{2} G\left(\theta^{x}, \theta^{y}\right)\right)^{2}} \frac{R}{\left(\left(R+\epsilon h\left(\theta^{x}\right)\right)^{2}+\left(\epsilon h^{\prime}\left(\theta^{x}\right)\right)^{2}\right)^{\frac{1}{2}}} R d \theta^{y},
\end{aligned}
$$

where $L_{\epsilon}:=-\frac{\langle\tilde{\nu}(\tilde{x}), \tilde{\nu}(\tilde{y})\rangle}{|\tilde{x}-\tilde{y}|^{2}}+2 \frac{\langle\tilde{x}-\tilde{y}, \tilde{\nu}(\tilde{x})\rangle\langle\tilde{x}-\tilde{y}, \tilde{\nu}(\tilde{y})\rangle}{|\tilde{x}-\tilde{y}|^{4}}$ is the kernel of $\mathcal{L}_{\epsilon}$ and the singularity of $\frac{N_{i}}{2 R^{4}(1-c)^{2}}$ for $i \in[0,4]$ is of order $O\left(\left|\theta^{x}-\theta^{y}\right|^{-2}\right)$. We do not give here the expressions of $N_{2}, N_{3}, N_{4}$ due to their length, but $N_{0}$ and $N_{1}$ are given by

$$
\begin{aligned}
& N_{0}=-R^{2}(1-c), \\
& N_{1}=-2 R(1-c)\left(h\left(\theta^{x}\right)+h\left(\theta^{y}\right)\right) .
\end{aligned}
$$


Recall that

$$
F\left(\theta^{x}, \theta^{y}\right)=\frac{\left(h\left(\theta^{x}\right)+h\left(\theta^{y}\right)\right)}{R}, \quad G\left(\theta^{x}, \theta^{y}\right)=\frac{\left(h\left(\theta^{x}\right)-h\left(\theta^{y}\right)^{2}+2 h\left(\theta^{x}\right) h\left(\theta^{y}\right)(1-c)\right)}{2 R^{2}(1-c)} .
$$

We introduce the following series, which converges absolutely and uniformly:

$$
\frac{1}{\left(1+\epsilon F\left(\theta^{x}, \theta^{y}\right)+\epsilon^{2} G\left(\theta^{x}, \theta^{y}\right)\right)^{2}} \frac{R}{\left(\left(R+\epsilon h\left(\theta^{x}\right)\right)^{2}+\left(\epsilon h^{\prime}\left(\theta^{x}\right)\right)^{2}\right)^{\frac{1}{2}}}=\sum_{p=0}^{\infty} \epsilon^{p} F_{p}\left(\theta^{x}, \theta^{y}\right) .
$$

The first-order term is given by

$$
F_{1}\left(\theta^{x}, \theta^{y}\right)=-\frac{\left(3 h\left(\theta^{x}\right)+2 h\left(\theta^{y}\right)\right)}{R} .
$$

Note that $\left(F_{p}\right)_{p \in \mathbb{N}}$, like $F$ and $G$, have no singularity and are uniformly bounded.

We define the following functions for all $x, y \in \partial C$ :

$$
\begin{aligned}
L^{(0)} & =\frac{N_{0}}{2 R^{4}(1-c)^{2}}, & L^{(1)} & =\frac{N_{0} F_{1}+N_{1}}{2 R^{4}(1-c)^{2}}, \\
L^{(2)} & =\frac{N_{0} F_{2}+N_{1} F_{1}+N_{2}}{2 R^{4}(1-c)^{2}}, & L^{(3)} & =\frac{N_{0} F_{3}+N_{1} F_{2}+N_{2} F_{1}+N_{3}}{2 R^{4}(1-c)^{2}},
\end{aligned}
$$

and, for $n \geq 4$,

$$
L^{(n)}=\frac{1}{2 R^{4}(1-c)^{2}}\left(N_{0} F_{n}+N_{1} F_{n-1}+N_{2} F_{n-2}+N_{3} F_{n-3}+N_{4} F_{n-4}\right) .
$$

Thanks to the explicit formulas of $\left(N_{i}\right)_{i \in[0,4]}$ and (4.19), we obtain in particular that, for all $x, y \in \partial C$,

$$
L^{(0)}=-\frac{1}{2 R^{3}(1-c)} \quad \text { and } \quad L^{(1)}=\frac{h\left(\theta^{x}\right)}{2 R^{3}(1-c)},
$$

where $c$ is given by (4.16).

By construction, $L^{(n)}$ for all $n \in \mathbb{N}$ have a singularity of order $O\left(\left|\theta^{x}-\theta^{y}\right|^{-2}\right)$.

The integral operators $\left(\mathcal{L}^{(n)}\right)_{n \in \mathbb{N}}$ associated to the kernels $\left(L^{(n)}\right)_{n \in \mathbb{N}}$ are given for all $f \in \mathcal{C}^{2, \eta}(\partial C), x \in \partial C$, by

$$
\mathcal{L}^{(n)}[f](x)=\frac{1}{2 \pi} \int_{0}^{2 \pi} L^{(n)}\left(\theta^{x}, \theta^{y}\right)\left(f\left(\theta^{y}\right)-f\left(\theta^{x}\right)\right) R d \theta^{y} .
$$

It follows from (4.21) that, for all $\mathcal{C}^{2, \eta}(\partial C), x \in \partial C$ :

$$
\mathcal{L}^{(0)}[f](x)=\mathcal{L}[f](x) \quad \text { and } \quad \mathcal{L}^{(1)}[f](x)=-h\left(\theta^{x}\right) \mathcal{L}[f](x) .
$$

We can now write, from our construction, an expansion of $\mathcal{L}_{\epsilon}$.

Proposition 4.3. Let $N \in \mathbb{N}$. There exists $C$ depending only on $\mathrm{R}$ and $\|h\|_{\mathcal{C}^{2}}$ such that, for any $\tilde{f} \in \mathcal{C}^{2, \eta}\left(\partial C_{\epsilon}\right), 0<\eta<1$, we have

$$
\left\|\mathcal{L}_{\epsilon}[\tilde{f}] \circ \tau_{\epsilon}-\mathcal{L}[f]-\sum_{n=0}^{N} \epsilon^{n} \mathcal{L}^{(n)}[f]\right\|_{\mathcal{C}^{1, \eta}(\partial C)} \leq C \epsilon^{N+1}\|f\|_{\mathcal{C}^{2, \eta}(\partial C)},
$$

where $\tau_{\epsilon}$ is the diffeomorphism from $\partial C$ onto $\partial C_{\epsilon}$ given by $\tau_{\epsilon}(x)=\tilde{x}$, and the function $f$ is defined by $f:=\tilde{f} \circ \tau_{\epsilon}$. 
Proof. Let $f \in \mathcal{C}^{2, \eta}$. We know that $\frac{N_{i}}{2 R^{4}(1-c)^{2}}$, for all $i \in[0,4]$, have a singularity of order $O\left(\left|\theta^{x}-\theta^{y}\right|^{-2}\right)$.

Thanks to the $\mathcal{C}^{1}$-character of $f,\left(\theta^{x}, \theta^{y}\right) \rightarrow \frac{N_{i}}{2 R^{4}(1-c)^{2}}\left(f\left(\theta^{y}\right)-f\left(\theta^{x}\right)\right)$ have a singularity of order $O\left(\left|\theta^{x}-\theta^{y}\right|^{-1}\right)$.

Besides, the Hilbert transform is a bounded operator from $\mathcal{C}^{0, \eta}$ to $\mathcal{C}^{0, \eta}$. From the boundedness of $h$ and its derivatives, it follows that the operators associated with the kernels $\frac{N_{i}}{2 R^{4}(1-c)^{2}}$ for $i \in[0,4]$ are bounded from $\mathcal{C}^{2, \eta}$ to $\mathcal{C}^{1, \eta}$.

Since the $\left(F_{p}\right)_{p \in \mathbb{N}}$ are uniformly bounded, the construction of $L^{(n)}$ (4.20) implies that there exists a constant $K\left(R,\|h\|_{\mathcal{C}^{2}}\right)$ such that

$$
\left\|\mathcal{L}^{(n)}[f]\right\|_{\mathcal{C}^{0, \eta}(\partial C)} \leq K\left\|f^{\prime}\right\|_{\mathcal{C}^{0, \eta}(\partial C)}
$$

where $f^{\prime}$ is the derivative of $f$ with respect to $\theta$. Likewise, since the kernel of $\mathcal{L}^{(n)}[f]^{\prime}(x)$ is of order $O\left(\frac{f(y)-f(x)-(x-y) f^{\prime}(x)}{|x-y|^{2}}\right)$, the $\mathcal{C}^{2}$-character of $f$ gives us a singularity of order $O\left(\left|\theta^{x}-\theta^{y}\right|^{-1}\right)$. We therefore obtain that

$$
\left\|\mathcal{L}^{(n)}[f]^{\prime}\right\|_{\mathcal{C}^{0, \eta}(\partial C)} \leq \tilde{K}\left\|f^{\prime \prime}\right\|_{\mathcal{C}^{0, \eta}(\partial C)},
$$

where $\tilde{K}\left(R,\|h\|_{\mathcal{C}^{2}}\right)$ is a constant and $f^{\prime \prime}$ is the second derivative of $f$. Therefore, there exists a constant $\widehat{K}\left(R,\|h\|_{\mathcal{C}^{2}}\right)$ such that

$$
\left\|\mathcal{L}^{(n)}[f]\right\|_{\mathcal{C}^{1, \eta}(\partial C)} \leq \widehat{K}\|f\|_{\mathcal{C}^{2, \eta}(\partial C)} .
$$

For all $n \in \mathbb{N}$, the operator $\mathcal{L}^{(n)}: \mathcal{C}^{2, \eta} \rightarrow \mathcal{C}^{1, \eta}$ is bounded, and the constant $\widehat{K}$ does not depend on $n$. Let $N \in \mathbb{N}$. Let $\tilde{f} \in \mathcal{C}^{2, \eta}\left(\partial C_{\epsilon}\right)$. We introduce $f:=\tilde{f} \circ \tau_{\epsilon}, f \in \mathcal{C}^{2, \eta}(\partial C)$. We have

$$
\left\|\sum_{n=N+1}^{\infty} \epsilon^{n} \mathcal{L}^{(n)}[f]\right\|_{\mathcal{C}^{1, \eta}(\partial C)} \leq \frac{\epsilon^{N+1}}{1-\epsilon} \widehat{K}\|f\|_{\mathcal{C}^{2, \eta}(\partial C)},
$$

which ends the proof of the result.

By substituting the result of Proposition 4.3 into the integral equation (4.9) verified by $\Psi_{\epsilon}$, we obtain for all $N \in \mathbb{N}$ that

$$
\forall x \in \partial C, \quad\left(I+\beta \mathcal{L}+\beta \sum_{n=0}^{N} \epsilon^{n} \mathcal{L}^{(n)}\right)\left[\Psi_{\epsilon}\right](\tilde{x})+o\left(\epsilon^{N}\right)=-\beta \frac{\partial H_{\epsilon}}{\partial \tilde{\nu}}(\tilde{x}) .
$$

We use Taylor-Lagrange's theorem and (4.13) to expand $\frac{\partial H_{\epsilon}}{\partial \tilde{\nu}}(\tilde{x})$ :

$$
\frac{\partial H_{\epsilon}}{\partial \tilde{\nu}}(\tilde{x})=\left(\sum_{p=0}^{\infty} \sum_{|\alpha|=p} \frac{\epsilon^{p}}{\alpha !}\left(\partial^{\alpha} \nabla H_{\epsilon}\right)(x)(h(\theta) \nu(x))^{\alpha}\right)\left(\sum_{p=0}^{\infty} \epsilon^{p} \nu^{(p)}(\theta)\right) .
$$

In particular, at first order, we have

$$
\frac{\partial H_{\epsilon}}{\partial \tilde{\nu}}(\tilde{x})=\frac{\partial H_{\epsilon}}{\partial r}(x)+\epsilon\left(-\frac{h^{\prime}(\theta)}{R^{2}} \frac{\partial H_{\epsilon}}{\partial \theta}(x)+h(\theta) \frac{\partial^{2} H_{\epsilon}}{\partial r^{2}}(x)\right) .
$$


Our integral equation (4.23) then becomes

$$
\forall x \in \partial C, \quad\left(I+\beta \mathcal{L}+\beta \sum_{p=0}^{N} \epsilon^{n} \mathcal{L}^{(n)}\right)\left[\Psi_{\epsilon}\right](\tilde{x})+o\left(\epsilon^{N}\right)=-\beta \sum_{n=0}^{\infty} \epsilon^{n} G_{n}(x),
$$

where $\left(G_{n}\right)_{n \in \mathbb{N}}$ are the coefficients in the expansion (4.24).

Equation (4.26) can therefore be solved recursively in the following way:

$$
\begin{aligned}
\Psi^{(0)} & =-\beta(I+\beta \mathcal{L})^{-1}\left[G_{0}\right] \\
\forall n \leq N, \quad \Psi^{(n)} & =-\beta(I+\beta \mathcal{L})^{-1}\left[G_{n}+\sum_{p=0}^{n-1} \mathcal{L}^{(n-p)} \Psi^{(p)}\right] .
\end{aligned}
$$

In particular, thanks to (4.22) and (4.25), we have

$$
\begin{aligned}
& \Psi^{(0)}=-\beta(I+\beta \mathcal{L})^{-1}\left(\frac{\partial H_{\epsilon}}{\partial \nu}\right), \\
& \Psi^{(1)}=-\beta(I+\beta \mathcal{L})^{-1}\left(-\frac{h^{\prime}}{R^{2}} \frac{\partial H_{\epsilon}}{\partial \theta}+h \frac{\partial^{2} H_{\epsilon}}{\partial r^{2}}-h \frac{\partial}{\partial \nu} \mathcal{D}_{C}^{(0)}\left[\Psi^{(0)}\right]\right) .
\end{aligned}
$$

We obtain the following proposition.

Proposition 4.4. Let $N \in \mathbb{N}$. There exists $K$, depending only on $N, R$ and the $\mathcal{C}^{2}$ norm of $h$, such that

$$
\left\|\Psi_{\epsilon}-\sum_{n=0}^{N} \epsilon^{n} \Psi^{(n)}\right\|_{\mathcal{C}^{2, \eta}(\partial C)} \leq K \epsilon^{N+1},
$$

where $\left(\Psi^{(n)}\right)_{n \leq N}$ are defined by the recursive relation (4.27).

In order to prove Proposition 4.4, we need the following result [20, Theorem 1.16].

Lemma 4.5. Let $X$ and $Y$ be two Banach spaces. Let $T$ and $A$ be two operators from $X$ to $Y$ such that $D(T) \subset D(A)$, where $D(T)$ and $D(A)$ are the domains of $T$ and $A$, respectively. Let $T^{-1}$ exist and be a bounded operator from $Y$ to $X$ (so that $T$ is closed). We suppose that two positive constants $a, b$ exist such that

$$
\begin{gathered}
\forall u \in D(T), \quad\|A u\| \leq a\|u\|+b\|T u\|, \\
a\left\|T^{-1}\right\|+b<1 .
\end{gathered}
$$

Then $S=T+A$ is closed and invertible, $S^{-1}$ is a bounded operator from $Y$ to $X$ and the following inequalities hold:

$$
\left\|S^{-1}\right\| \leq \frac{\left\|T^{-1}\right\|}{1-a\left\|T^{-1}\right\|-b}, \quad\left\|S^{-1}-T^{-1}\right\| \leq \frac{\left\|T^{-1}\right\|\left(a\left\|T^{-1}\right\|+b\right)}{1-a\left\|T^{-1}\right\|-b} .
$$

If in addition $T^{-1}$ is compact, then so is $S^{-1}$.

Proof of Proposition 4.4. By definition, $\Psi_{\epsilon}$ verifies:

$$
\left(I+\beta \mathcal{L}_{\epsilon}\right)\left[\Psi_{\epsilon}\right]=-\beta \sum_{n=0}^{\infty} \epsilon^{n} G_{n}
$$


Besides, it follows from our recursive construction of the $\left(\Psi^{(i)}\right)_{i \in[0, N]}$ that

$$
\left(I+\beta \mathcal{L}+\beta \sum_{n=1}^{N} \epsilon^{n} \mathcal{L}^{(n)}\right)\left[\sum_{n=0}^{N} \epsilon^{p} \Psi^{(p)}\right]=-\beta \sum_{n=0}^{\infty} \epsilon^{n} G_{n}+\epsilon^{N+1} A_{N},
$$

where $A_{N}=\sum_{n=0}^{N} \epsilon^{n} \sum_{p=0}^{N+n} \mathcal{L}^{(N+1+n-p)}\left[\Psi^{(p)}\right]+\beta \sum_{n=0}^{\infty} \epsilon^{n} G_{N+1+n}$.

Therefore, we have

$$
\begin{aligned}
\Psi_{\epsilon}-\sum_{n=0}^{N} \epsilon^{n} \Psi^{(n)}= & \left(\left(I+\beta \mathcal{L}_{\epsilon}\right)^{-1}-\left(I+\beta \mathcal{L}+\beta \sum_{n=1}^{N} \epsilon^{n} \mathcal{L}^{(n)}\right)^{-1}\right)\left[-\beta \sum_{n=0}^{\infty} \epsilon^{n} G_{n}\right] \\
& -\left(I+\beta \mathcal{L}+\beta \sum_{n=1}^{N} \epsilon^{n} \mathcal{L}^{(n)}\right)^{-1}\left[\epsilon^{N+1} A_{N}\right] .
\end{aligned}
$$

We know from Proposition 4.2 that the bounded operator $T:=I+\beta \mathcal{L}_{\epsilon}: \mathcal{C}^{2, \eta} \rightarrow \mathcal{C}^{1, \eta}$ has a bounded inverse $T^{-1}: \mathcal{C}^{1, \eta} \rightarrow \mathcal{C}^{2, \eta}$. We define

$$
A:=\beta \mathcal{L}+\beta \sum_{n=1}^{N} \epsilon^{n} \mathcal{L}^{(n)}-\beta \mathcal{L}_{\epsilon} .
$$

From Proposition 4.3, it follows that there exists a constant $C\left(R,\|h\|_{\mathcal{C}^{2}}\right)$ such that

$$
\|A[u]\|_{\mathcal{C}^{1, \eta}(\partial C)} \leq C \epsilon^{N+1}\|u\|_{\mathcal{C}^{2, \eta}(\partial C)} .
$$

For $\epsilon$ small enough, we have

$$
C \epsilon^{N+1}\left\|T^{-1}\right\|<1 .
$$

In the following, we apply Lemma 4.5 with $a:=C \epsilon^{N+1}$ and $b:=0$.

The operator $S:=I+\beta \mathcal{L}+\beta \sum_{n=1}^{N} \epsilon^{n} \mathcal{L}^{(n)}$ has a bounded inverse, which satisfies

$$
\left\|\left(I+\beta \mathcal{L}+\beta \sum_{n=1}^{N} \epsilon^{n} \mathcal{L}^{(n)}\right)^{-1}\right\| \leq \frac{\left\|T^{-1}\right\|}{1-C \epsilon^{N+1}\left\|T^{-1}\right\|}
$$

and $\quad\left\|\left(I+\beta \mathcal{L}+\beta \sum_{n=1}^{N} \epsilon^{n} \mathcal{L}^{(n)}\right)^{-1}-\left(I+\beta \mathcal{L}_{D}\right)^{-1}\right\| \leq \frac{C \epsilon^{N+1}\left\|T^{-1}\right\|^{2}}{1-C \epsilon^{N+1}\left\|T^{-1}\right\|}$.

We use (4.30) to get

$$
\left\|\Psi_{\epsilon}-\sum_{n=0}^{N} \epsilon^{n} \Psi^{(n)}\right\|_{\mathcal{C}^{2, \eta}} \leq \frac{\epsilon^{N+1}\left\|T^{-1}\right\|}{1-C \epsilon^{N+1}\left\|T^{-1}\right\|}\left(C\left\|T^{-1}\right\|\left\|\beta \frac{\partial H_{\epsilon}}{\partial \tilde{\nu}}\right\|_{\mathcal{C}^{1, \eta}}+\left\|A_{N}\right\|_{\mathcal{C}^{1, \eta}}\right) .
$$

Recall that $H_{\epsilon}$ is $\mathcal{C}^{\infty}$ on $\partial C$. Hence, for all $p \in \mathbb{N}, G_{p}$ is bounded. From Proposition 4.3. we know that $\mathcal{L}^{(n)}: \mathcal{C}^{2, \eta}(\partial C) \rightarrow \mathcal{C}^{1, \eta}(\partial C)$, for all $n \in \mathbb{N}$, are bounded operators. We have also from Proposition 4.2 that $(I+\beta \mathcal{L})^{-1}: \mathcal{C}^{1, \eta}(\partial C) \rightarrow \mathcal{C}^{2, \eta}(\partial C)$ is bounded. One can prove recursively from the construction (4.29) that, for all $p \in \mathbb{N}, \Psi^{(p)}$ is $\mathcal{C}^{2, \eta}(\partial C)$ bounded. $A_{N}$ and $\frac{\partial H_{\epsilon}}{\partial \tilde{\nu}}$ are therefore $\mathcal{C}^{1, \eta}(\partial C)$-bounded. 
Finally, we obtain that there exists a constant $K\left(N, R,\|h\|_{\mathcal{C}^{2}}\right)$ such that

$$
\left\|\Psi_{\epsilon}-\sum_{n=0}^{N} \epsilon^{n} \Psi^{(n)}\right\|_{\mathcal{C}^{2, \eta}} \leq K \epsilon^{N+1},
$$

and the proof of Proposition 4.4 is complete.

We now define the first-order term in the expansion of $\widetilde{c_{f l r}}$ as a function of the cell membrane perturbation. For doing so, we introduce, for $n \in \mathbb{N} \backslash\{0\}$ and $x \in \partial \Omega$,

$$
v_{n}(x):=\sum_{i+j+k+l=n} \int_{0}^{2 \pi} \frac{h(y)^{i}}{i !}\left(\nabla_{y}\left(\frac{\partial^{i}}{\partial r_{y}^{i}} \Gamma^{(0)}(x, y)\right) \cdot \nu^{(j)}(y)\right) \Psi^{(k)}\left(\theta^{y}\right) \sigma^{(l)}\left(\theta^{y}\right) d \theta^{y} .
$$

It follows from (4.8), (4.14), (4.29) and (4.31) that for all $x \in \partial \Omega$,

$$
\begin{aligned}
v_{1}(x) & =\int_{0}^{2 \pi} \frac{\partial^{2}}{\partial r_{y}^{2}} \Gamma^{(0)}(x, y) h\left(\theta^{y}\right) \Psi^{(0)}\left(\theta^{y}\right) R d \theta^{y}-\frac{1}{R} \int_{0}^{2 \pi} \frac{\partial}{\partial \theta^{y}} \Gamma^{(0)}(x, y) \Psi^{(0)}\left(\theta^{y}\right) h^{\prime}\left(\theta^{y}\right) d \theta^{y} \\
& +\int_{0}^{2 \pi} \frac{\partial}{\partial r_{y}} \Gamma^{(0)}(x, y) \Psi^{(1)}\left(\theta^{y}\right) R d \theta^{y} \quad+\int_{0}^{2 \pi} \frac{\partial}{\partial r_{y}} \Gamma^{(0)}(x, y) \Psi^{(0)}\left(\theta^{y}\right) h\left(\theta^{y}\right) d \theta^{y}
\end{aligned}
$$

In terms of polar coordinates, the Laplacian has the following expression:

$$
\Delta=\frac{\partial^{2}}{\partial r^{2}}+\frac{1}{r} \frac{\partial}{\partial r}+\frac{1}{r^{2}} \frac{\partial^{2}}{\partial \theta^{2}}
$$

Therefore, we have for all $x \in \partial \Omega$ :

$$
\begin{aligned}
v_{1}(x)= & -\frac{1}{R} \int_{0}^{2 \pi} \frac{\partial^{2}}{\partial \theta^{y^{2}}} \Gamma^{(0)}(x, y) h\left(\theta^{y}\right) \Psi^{(0)}\left(\theta^{y}\right) d \theta^{y} \\
& -\frac{1}{R} \int_{0}^{2 \pi} \frac{\partial}{\partial \theta^{y}} \Gamma^{(0)}(x, y) \Psi^{(0)}\left(\theta^{y}\right) h^{\prime}\left(\theta^{y}\right) d \theta^{y} \\
& +\int_{0}^{2 \pi} \frac{\partial}{\partial r_{y}} \Gamma^{(0)}(x, y) \Psi^{(1)}\left(\theta^{y}\right) R d \theta^{y} .
\end{aligned}
$$

Besides, we obtain, thanks to (4.29) and (4.31), that

$$
\mathcal{D}_{C_{\epsilon}}^{(0)}\left[\Psi_{\epsilon}\right](x)=-\beta \mathcal{D}_{C}^{(0)}(I+\beta \mathcal{L})^{-1}\left[\left.\frac{\partial H_{\epsilon}}{\partial \nu}\right|_{\partial C}\right]+\sum_{n=1}^{N} \epsilon^{n} v_{n}(x)+o\left(\epsilon^{N}\right) .
$$

The integral equation (4.10) that $\Psi$ verifies then gives us

$$
\mathcal{D}_{C_{\epsilon}}^{(0)}\left[\Psi_{\epsilon}\right]-\mathcal{D}_{C}^{(0)}[\Psi]=-\beta \mathcal{D}_{C}^{(0)}(I+\beta \mathcal{L})^{-1}\left[\left.\frac{\partial H_{\epsilon}}{\partial \nu}\right|_{\partial C}-\left.\frac{\partial H}{\partial \nu}\right|_{\partial C}\right]+\sum_{n=1}^{N} \epsilon^{n} v_{n}+o\left(\epsilon^{N}\right) .
$$

By definition, we have on $\partial C$

$$
H_{\epsilon}-H=\mathcal{D}_{\Omega}^{(0)}\left[\left.u_{\epsilon}\right|_{\partial \Omega}-\left.u\right|_{\partial \Omega}\right] .
$$

Let $\mathcal{E}$ be the operator defined by

$$
\mathcal{E}[v](x):=\beta \mathcal{D}_{C}^{(0)}(I+\beta \mathcal{L})^{-1}\left[\left.\frac{\partial}{\partial \nu}\left(\mathcal{D}_{\Omega}^{(0)} v\right)\right|_{\partial C}\right](x)-\left(\frac{I}{2}+\mathcal{K}_{\Omega}^{(0)}\right)[v](x),
$$

for all $v \in L_{0}^{2}(\partial \Omega)$ and $x \in \partial \Omega$. 
Recall that on $\partial \Omega$ :

$$
u_{\epsilon}(x)-u(x)=\left(\frac{I}{2}+\mathcal{K}_{\Omega}^{(0)}\right)\left[u_{\epsilon}-u\right](x)+\mathcal{D}_{C_{\epsilon}}^{(0)}\left[\Psi_{\epsilon}\right](x)-\mathcal{D}_{C}^{(0)}[\Psi](x) .
$$

We obtain for all $x \in \partial \Omega$ that

$$
(I+\mathcal{E})\left[u_{\epsilon}-u\right](x)=\sum_{n=1}^{N} \epsilon^{n} v_{n}(x)+o\left(\epsilon^{N}\right),
$$

and, at first order,

$$
(I+\mathcal{E})\left[u_{\epsilon}-u\right](x)=\epsilon v_{1}(x)+o(\epsilon),
$$

where $v_{1}$ is given by the formula

$$
v_{1}(x)=-\frac{1}{R} \int_{0}^{2 \pi} \frac{\partial}{\partial \theta^{y}}\left(h\left(\theta^{y}\right) \frac{\partial}{\partial \theta^{y}} \Gamma^{(0)}(x, y)\right) \Psi^{(0)}\left(\theta^{y}\right) d \theta^{y}+\mathcal{D}_{C}^{(0)}\left[\Psi^{(1)}\right](x) .
$$

Proposition 4.6. Let $\mathcal{E}$ be defined by (4.32). The operator $I+\mathcal{E}$ is invertible on $L_{0}^{2}(\partial \Omega)$.

Proof. The operator $\mathcal{E}$ is compact. We can therefore apply the Fredholm alternative. Let us prove the injectivity of $I+\mathcal{E}$. For doing so, we introduce the function $v$ defined on $\Omega$ by

$$
v(x)=\mathcal{D}_{\Omega}^{(0)}\left[\left.v\right|_{\partial \Omega}\right]-\beta \mathcal{D}_{C}^{(0)}(I+\beta \mathcal{L})^{-1}\left[\left.\frac{\partial}{\partial \nu}\left(\mathcal{D}_{\Omega}^{(0)}[v]\right)\right|_{\partial C}\right] .
$$

It follows from Proposition 3.5 that $v$ is solution to (2.4) with $H=\mathcal{D}_{\Omega}^{(0)}\left[\left.v\right|_{\partial \Omega}\right]$. The decomposition of the representation formula of such a solution is unique so that we have $\mathcal{S}_{\Omega}^{(0)}\left[\left.\frac{\partial v}{\partial \nu}\right|_{\partial \Omega}\right]=0$ and hence $\left.\frac{\partial v}{\partial \nu}\right|_{\partial \Omega}=0$. Since $v$ is harmonic, we obtain that $v$ is constant in $\Omega$. Recall that $\int_{\partial \Omega} v=0$. Therefore, we have $v=0$ in $\Omega$. Besides, on $\partial \Omega, v$ verifies

$$
\forall x \in \partial \Omega, \quad v(x)=-\mathcal{E}[v](x) .
$$

We have proved the injectivity and hence the invertibility of $I+\mathcal{E}$ on $L_{0}^{2}(\partial \Omega)$.

Now, combining Proposition 4.6 and (4.33) yields

$$
u_{\epsilon}(x)-u(x)=\sum_{n=1}^{N} \epsilon^{n}(I+\mathcal{E})^{-1}\left[v_{n}\right](x)+o\left(\epsilon^{N}\right) .
$$

Note that, by construction, $\Psi^{(n)}$ and thus $v_{n}$ still depend on $\epsilon$. We can remove this dependance from our asymptotic formula in the following way. We introduce $\left(G_{n}^{0}\right)_{n \in \mathbb{N}}$, the expansion of $\frac{\partial H}{\partial \tilde{\nu}}$. Let $\left(v_{n}^{0}\right)_{n \in \mathbb{N} \backslash\{0\}}$ and $\left(\Psi_{0}^{(n)}\right)_{n \in \mathbb{N}}$ be defined by (4.31) and (4.27), where $\left(G_{n}\right)_{n \in \mathbb{N}}$ is replaced respectively by $\left(G_{n}^{0}\right)_{n \in \mathbb{N}}$. We then obtain that

$$
\begin{aligned}
& \forall x \in \partial C, \quad \Psi_{\epsilon}(x)=\Psi_{0}^{(0)}(x)+o(1), \\
& \forall x \in \partial \Omega, \quad u_{\epsilon}(x)=u(x)+o(1) .
\end{aligned}
$$


By repeating the same procedure with $H+\epsilon \mathcal{D}_{\Omega}^{(0)}(I+\mathcal{E})^{-1}\left[v_{1}^{0}\right]$ instead of $H$, one finds $\left(v_{n}^{1}\right)_{n \in \mathbb{N}^{*}}$ and $\left(\Psi_{1}^{(n)}\right)_{n \in \mathbb{N}}$ such that

$$
\begin{aligned}
& \forall x \in \partial C, \quad \Psi_{\epsilon}(x)=\Psi_{1}^{(0)}(x)+\epsilon \Psi_{1}^{(1)}(x)+o(\epsilon), \\
& \forall x \in \partial \Omega, \quad u_{\epsilon}(x)=u(x)+\epsilon(I+\mathcal{E})^{-1}\left[v_{1}^{1}\right]+o(\epsilon) .
\end{aligned}
$$

One can prove the following proposition by repeating the same procedure until one obtains $\left(v_{n}^{N}\right)_{n \in \mathbb{N} \backslash\{0\}}$.

Proposition 4.7. Let $\left(v_{n}^{N}\right)_{n \in[1, N]}$ and $\left(\Psi_{N}^{(n)}\right)_{n \in[0, N]}$ be the functions defined above. The following asymptotic formulas hold:

$$
\begin{aligned}
& \forall x \in \partial C, \quad \Psi_{\epsilon}(x)=\sum_{n=1}^{N} \epsilon^{n} \Psi_{N}^{(n)}+o\left(\epsilon^{N}\right), \\
& \forall x \in \partial \Omega, \quad u_{\epsilon}(x)-u(x)=\sum_{n=1}^{N} \epsilon^{n}(I+\mathcal{E})^{-1}\left[v_{n}^{N}\right](x)+o\left(\epsilon^{N}\right) .
\end{aligned}
$$

The remainder $o\left(\epsilon^{N}\right)$ depends only on $N, R$ and $\|h\|_{\mathcal{C}^{2}}$.

We can now compute the first-order term in the expansion of $\widetilde{c_{f f r}}$.

Recall that $\widetilde{c_{\mathrm{fr}}}=\left.\delta\left[u_{\epsilon}\right]\right|_{\partial C_{\epsilon}}$. The boundary conditions (2.4) that $u_{\epsilon}$ satisfies give us

$$
\widetilde{c_{\mathrm{fr}}}=\delta \beta \frac{\partial u_{\epsilon}}{\partial \nu}=-\delta \Psi_{\epsilon} .
$$

Let us find the first-order approximation of $\Psi_{\epsilon}$. We apply the previous procedure to obtain $\Psi_{1}^{(1)}$. Hence, one introduces

$$
\begin{aligned}
& \Psi_{0}^{(0)}=-\beta(I+\beta \mathcal{L})^{-1}\left[\frac{\partial H}{\partial \nu}\right] \\
& \Psi_{0}^{(1)}=-\beta(I+\beta \mathcal{L})^{-1}\left[-\frac{h^{\prime}}{R^{2}} \frac{\partial H}{\partial \theta}+h \frac{\partial^{2} H}{\partial r^{2}}-h \frac{\partial}{\partial r} \mathcal{D}_{C}^{(0)}\left(\Psi_{0}^{(0)}\right)\right] .
\end{aligned}
$$

Observe that $\Psi_{0}^{(0)}=\Psi$. Thanks to (4.34), one can write $v_{1}^{0}$ for all $x \in \partial \Omega$ :

$$
v_{1}^{0}(x)=-\frac{1}{R} \int_{0}^{2 \pi} \frac{\partial}{\partial \theta^{y}}\left(h\left(\theta^{y}\right) \frac{\partial}{\partial \theta^{y}} \Gamma^{(0)}(x, y)\right) \Psi\left(\theta^{y}\right) d \theta^{y}+\mathcal{D}_{C}^{(0)}\left[\Psi_{0}^{(1)}\right](x) .
$$

Therefore, we get

$$
\begin{aligned}
& \Psi_{1}^{(0)}=\Psi_{0}^{(0)}=\Psi \\
& \Psi_{1}^{(1)}=-\beta(I+\beta \mathcal{L})^{-1}\left(-\frac{h^{\prime}}{R^{2}} \frac{\partial H}{\partial \theta}+h \frac{\partial^{2} H}{\partial r^{2}}+\frac{\partial}{\partial r} \mathcal{D}_{\Omega}^{(0)}(I+\mathcal{E})^{-1}\left[v_{1}^{0}\right]-h \frac{\partial}{\partial r} \mathcal{D}_{C}^{(0)}[\Psi]\right) .
\end{aligned}
$$

We first recall the mapping properties of the operators $\mathcal{K}_{D}^{(0)}$ and $\left(\mathcal{K}_{D}^{(0)}\right)^{*}$. It is known that if $D$ is a $\mathcal{C}^{2, \eta}$ domain, then $\mathcal{K}_{D}^{(0)}$ and $\left(\mathcal{K}_{D}^{(0)}\right)^{*}$ map continuously $\mathcal{C}^{1, \eta}(\partial D)$ into $\mathcal{C}^{2, \eta}(\partial D)$ (see, for instance, [39]). We also need the following result. 
Lemma 4.8. Let $D$ be a $\mathcal{C}^{2, \eta}$ domain in $\mathbb{R}^{2}$ for $0<\eta<1$. Let $\Psi \in \mathcal{C}^{1, \eta}(\partial D)$. We have

$$
\left.\frac{\partial}{\partial T} \mathcal{D}_{D}^{(0)}[\Psi]\right|_{ \pm}=\mp \frac{1}{2} \frac{\partial \Psi}{\partial T}+\frac{\partial}{\partial T} \mathcal{K}_{D}^{(0)}[\Psi] .
$$

Proof. Let $\Psi \in \mathcal{C}^{1, \eta}(\partial D)$. Recall the jump relation of the double layer potential across the boundary $\partial D$ :

$$
\left.\mathcal{D}_{D}^{(0)}[\Psi]\right|_{ \pm}=\left(\mp \frac{I}{2}+\mathcal{K}_{D}^{(0)}\right)[\Psi]
$$

The result of the proposition is simply obtained by taking the tangential derivative of the previous formula and making use of the mapping properties of $\mathcal{K}_{D}^{(0)}$.

Corollary 4.9. Let $D$ be a $\mathcal{C}^{2, \eta}$ domain in $\mathbb{R}^{2}$ for $0<\eta<1$. Let $h \in \mathcal{C}^{2}(\partial D)$, and let $\Psi \in \mathcal{C}^{2, \eta}(\partial D)$. We have

$$
-\left.\frac{\partial}{\partial T} h \frac{\partial}{\partial T} \mathcal{D}_{D}^{(0)}[\Psi]\right|_{-}+\left(-\frac{I}{2}+\left(\mathcal{K}_{D}^{(0)}\right)^{*}\right)\left[-\frac{\partial}{\partial T} h \frac{\partial \Psi}{\partial T}\right]=\frac{\partial}{\partial T} \mathcal{K}_{D}^{(0)}\left[h \frac{\partial \Psi}{\partial T}\right]-\frac{\partial}{\partial T} h \frac{\partial}{\partial T} \mathcal{K}_{D}^{(0)}[\Psi] .
$$

In the particular case of the disk $C$, we obtain that

$$
-\left.\frac{1}{R^{2}} \frac{\partial}{\partial \theta} h \frac{\partial}{\partial \theta} \mathcal{D}_{C}^{(0)}[\Psi]\right|_{-}+\left(-\frac{I}{2}+\left(\mathcal{K}_{C}^{(0)}\right)^{*}\right)\left[-\frac{1}{R^{2}} \frac{\partial}{\partial \theta} h \frac{\partial \Psi}{\partial \theta}\right]=0 .
$$

Proof. From Lemma 4.8, we know that

$$
-\left.\frac{\partial}{\partial T} h \frac{\partial}{\partial T} \mathcal{D}_{D}^{(0)}[\Psi]\right|_{-}=-\frac{1}{2} \frac{\partial}{\partial T} h \frac{\partial \Psi}{\partial T}-\frac{\partial}{\partial T} h \frac{\partial}{\partial T} \mathcal{K}_{D}^{(0)}[\Psi] .
$$

Besides, the tangential derivative of the operator $\mathcal{K}_{D}^{(0)}$ can be expressed as follows [22, p.144]:

$$
\frac{\partial}{\partial T} \mathcal{K}_{D}^{(0)}[\Psi]=-\left(\mathcal{K}_{D}^{(0)}\right)^{*}\left[\frac{\partial \Psi}{\partial T}\right]
$$

for $\Psi \in \mathcal{C}^{2, \eta}(\partial D)$. We thus obtain easily the result (4.38).

Recall that, for a disk of radius $R$, the operator $\mathcal{K}_{C}^{(0)}$ admits the explicit formula

$$
\mathcal{K}_{C}^{(0)}[\Psi]=\frac{1}{4 \pi} \int_{0}^{2 \pi} \Psi(\phi) d \phi,
$$

which does not depend on $\theta$. Its tangential derivative is therefore zero, and we have the formula for the disk. Finally, we note that $\left(\mathcal{K}_{C}^{(0)}\right)^{*}=\mathcal{K}_{C}^{(0)}$ and hence,

$$
\left(\mathcal{K}_{C}^{(0)}\right)^{*}\left[\frac{\partial}{\partial \theta} h \frac{\partial \Psi}{\partial \theta}\right]=0
$$

The next step is to find $w$ such that

$$
(I+\mathcal{E})[w]=v_{1}^{0} .
$$

From Proposition 4.6, it follows that there exists a unique function $w$ solution to (4.39). The following result holds. 
Proposition 4.10. The solution to (4.39) verifies the following equation and boundary conditions:

$$
\begin{cases}\Delta w=0 & \text { in } C \cup \Omega \backslash \bar{C} \\ \left.\frac{\partial w}{\partial \nu}\right|_{+}-\left.\frac{\partial w}{\partial \nu}\right|_{-}=-\frac{1}{R^{2}} \frac{\partial}{\partial \theta} h \frac{\partial \Psi}{\partial \theta} & \text { on } \partial C, \\ \left.w\right|_{+}-\left.w\right|_{-}-\left.\beta \frac{\partial w}{\partial \nu}\right|_{-}=-\beta\left(\frac{h}{R} \frac{\partial u}{\partial r}+\left.\frac{1}{R^{2}} \frac{\partial}{\partial \theta} h \frac{\partial u}{\partial \theta}\right|_{-}\right) & \text {on } \partial C, \\ \left.\frac{\partial w}{\partial \nu}\right|_{\partial \Omega}=0 & \text { on } \partial \Omega .\end{cases}
$$

Proof. The solution $w$ of the problem (4.40) satisfies the representation formula

$$
\forall x \in \Omega, \quad w(x)=\mathcal{D}_{\Omega}^{(0)}\left[\left.w\right|_{\partial \Omega}\right](x)+\mathcal{S}_{C}^{(0)}\left[-\frac{1}{R^{2}} \frac{\partial}{\partial \theta} h \frac{\partial \Psi}{\partial \theta}\right](x)+\mathcal{D}_{C}^{(0)}[\Lambda](x),
$$

where the density $\Lambda$ on $\partial C$ is given by

$$
\begin{aligned}
\Lambda=-\beta(I+\beta \mathcal{L})^{-1}\left[-\frac{h}{R} \frac{\partial u}{\partial r}-\left.\frac{1}{R^{2}} \frac{\partial}{\partial \theta} h \frac{\partial u}{\partial \theta}\right|_{-}+\frac{\partial}{\partial \nu} \mathcal{D}_{\Omega}^{(0)}\left[\left.w\right|_{\partial \Omega}\right]\right. \\
\left.+\left(-\frac{I}{2}+\left(\mathcal{K}_{C}^{*}\right)^{(0)}\right)\left[-\frac{1}{R^{2}} \frac{\partial}{\partial \theta} h \frac{\partial \Psi}{\partial \theta}\right]\right] .
\end{aligned}
$$

Thus, for $x \in \partial \Omega$,

$$
\begin{aligned}
& (I+\mathcal{E})[w](x)=\mathcal{S}_{C}^{(0)}\left[-\frac{1}{R^{2}} \frac{\partial}{\partial \theta} h \frac{\partial \Psi}{\partial \theta}\right](x) \\
& \quad-\beta \mathcal{D}_{C}^{(0)}(I+\beta \mathcal{L})^{-1}\left[-\frac{h}{R} \frac{\partial u}{\partial r}-\left.\frac{1}{R^{2}} \frac{\partial}{\partial \theta} h \frac{\partial u}{\partial \theta}\right|_{-}+\left(-\frac{I}{2}+\left(\mathcal{K}_{C}^{(0)}\right)^{*}\right)\left[-\frac{1}{R^{2}} \frac{\partial}{\partial \theta} h \frac{\partial \Psi}{\partial \theta}\right]\right]
\end{aligned}
$$

By integrating by parts twice, the first term in our equation becomes

$$
\mathcal{S}_{C}^{(0)}\left[-\frac{1}{R^{2}} \frac{\partial}{\partial \theta} h \frac{\partial \Psi}{\partial \theta}\right](x)=-\frac{1}{R} \int_{0}^{2 \pi} \frac{\partial}{\partial \theta^{y}}\left(h\left(\theta^{y}\right) \frac{\partial}{\partial \theta^{y}} \Gamma^{(0)}(x, y)\right) \Psi\left(\theta^{y}\right) d \theta^{y} .
$$

Hence, we obtain that

$$
\mathcal{S}_{C}^{(0)}\left[-\frac{1}{R^{2}} \frac{\partial}{\partial \theta} h \frac{\partial \Psi}{\partial \theta}\right](x)=v_{1}^{1}(x)-\mathcal{D}_{C}^{(0)}\left[\Psi_{1}^{(1)}\right](x) .
$$

The representation formula of $u$ and the expression of the Laplacian in terms of polar coordinates give us

$$
\left.\frac{1}{R^{2}} \frac{\partial}{\partial \theta} h \frac{\partial u}{\partial \theta}\right|_{-}=\frac{h^{\prime}}{R^{2}} \frac{\partial H}{\partial \theta}-h \frac{\partial^{2} H}{\partial r^{2}}-\frac{h}{R} \frac{\partial H}{\partial r}+\left.\frac{1}{R^{2}} \frac{\partial}{\partial \theta} h \frac{\partial}{\partial \theta} \mathcal{D}_{C}^{(0)}[\Psi]\right|_{-} .
$$

Observe that by definition of $\Psi$, we have on $\partial \Omega$

$$
\frac{\partial u}{\partial r}=-\beta^{-1} \Psi
$$


One can then derive the integral equation that $\Psi$ verifies and obtain that

$$
-\frac{h}{R} \frac{\partial u}{\partial r}+\frac{h}{R} \frac{\partial H}{\partial r}=-\frac{h}{R} \frac{\partial}{\partial r} \mathcal{D}_{C}^{(0)}[\Psi] .
$$

The second term in our equation (4.43) becomes

$$
-\beta \mathcal{D}_{C}^{(0)}(I+\beta \mathcal{L})^{-1}\left[-\frac{h^{\prime}}{R^{2}} \frac{\partial H}{\partial \theta}+h \frac{\partial^{2} H}{\partial r^{2}}-\frac{h}{R} \frac{\partial}{\partial r} \mathcal{D}_{C}^{(0)}(\Psi)\right] .
$$

It follows from (4.37) and (4.45) that

$$
\forall x \in \partial \Omega, \quad(I+\mathcal{E})[w](x)=v_{1}^{0}(x) .
$$

We have obtained an approximation at first order of $\widetilde{c_{\mathrm{fl}}}$ :

$$
\widetilde{c_{\mathrm{flr}}}=c_{\mathrm{flr}}-\epsilon \delta \Psi_{1}^{(1)}+o(\epsilon)
$$

where $\Psi_{1}^{(1)}$ is given by

$$
\Psi_{1}^{(1)}=-\beta(I+\beta \mathcal{L})^{-1}\left[-\frac{h^{\prime}}{R^{2}} \frac{\partial H}{\partial \theta}+h \frac{\partial^{2} H}{\partial r^{2}}+\frac{\partial}{\partial r} \mathcal{D}_{\Omega}^{(0)} w-h \frac{\partial}{\partial r} \mathcal{D}_{C}^{(0)}(\Psi)\right]
$$

and $w$ is the solution of (4.40).

We can now derive the first-order term in the asymptotic expansion of (4.4) as $\epsilon \rightarrow 0$.

ThEOREM 4.11. The integral (4.4) admits the following asymptotic expansion:

$$
\begin{aligned}
& \int_{\partial C_{\epsilon}} \tilde{\gamma} \widetilde{c_{\mathrm{fr}}}(x) \Phi_{\mathrm{exc}}^{n}(x) \Phi_{\mathrm{exc}}^{m}(x) d s(x)=\int_{\partial C} \tilde{\gamma} c_{\mathrm{flr}}(x) \Phi_{\mathrm{exc}}^{n}(x) \Phi_{\mathrm{exc}}^{m}(x) d s(x) \\
&+\epsilon \int_{\partial C} \tilde{\gamma}\left(A c_{\mathrm{frr}}(\theta) h(\theta)-\delta B \Psi_{1}^{(1)}(\theta)\right) e^{-i(n+m) \theta} d \theta+o(\epsilon),
\end{aligned}
$$

where the constants $A$ and $B$ are given by

$$
\begin{aligned}
& A=i k J_{n}^{\prime}(i k R) J_{m}(i k R) R+i k J_{n}(i k R) J_{m}^{\prime}(i k R) R+J_{n}(i k R) J_{m}(i k R), \\
& B=J_{n}(i k R) J_{m}(i k R) R .
\end{aligned}
$$

4.2.2. Fourier coefficients of $\Psi_{1}^{(1)}$. Recall that $\Omega$ is the unit disk and $C$ is the disk with radius $R<1$. In terms of polar coordinates, the fundamental solution $\Gamma^{(0)}$ of $\Delta$ in $\mathbb{R}^{2}$, given by (3.9), has the expression

$$
\forall y(r, \theta) \in \bar{\Omega}, \forall z(R, \phi) \in \bar{\Omega}, \quad \Gamma_{z}^{0}(y)=\frac{1}{4 \pi} \log \left(R^{2}+r^{2}-2 r R \cos (\theta-\phi)\right) .
$$

The decomposition of log into a power series gives us the following formulas:

$$
\Gamma_{z}^{0}(y)= \begin{cases}\frac{1}{2 \pi} \log R-\frac{1}{4 \pi} \sum_{n \in \mathbb{Z}^{*}} \frac{1}{|n|}\left(\frac{r}{R}\right)^{|n|} e^{i n(\theta-\phi)} & \text { if } r<R \\ \frac{1}{2 \pi} \log r-\frac{1}{4 \pi} \sum_{n \in \mathbb{Z}^{*}} \frac{1}{|n|}\left(\frac{R}{r}\right)^{|n|} e^{i n(\theta-\phi)} & \text { if } R<r\end{cases}
$$

where $\mathbb{Z}^{*}=\mathbb{Z} \backslash\{0\}$. 
Let $f \in L^{2}(] 0,2 \pi[)$. By reinjecting (4.51) into the definition of the following operators, we obtain for $y(R, \theta) \in \partial C$ that

$$
\begin{aligned}
\mathcal{S}_{\Omega}^{(0)}[f](y) & =-\frac{1}{2} \sum_{n \in \mathbb{Z}^{*}} \frac{1}{|n|} R^{|n|} \hat{f}(n) e^{i n \theta}, \\
\mathcal{D}_{\Omega}^{(0)}[f](y) & =\hat{f}(0)+\frac{1}{2} \sum_{n \in \mathbb{Z}^{*}} R^{|n|} \hat{f}(n) e^{i n \theta}, \\
\frac{\partial \mathcal{D}_{\Omega}^{(0)}}{\partial r}[f](y) & =\frac{1}{2} \sum_{n \in \mathbb{Z}^{*}}|n| R^{|n|-1} \hat{f}(n) e^{i n \theta}, \\
\frac{\partial \mathcal{D}_{C}^{(0)}}{\partial r}[f](y) & =\frac{1}{2} \sum_{n \in \mathbb{Z}^{*}}|n| \frac{1}{R} \hat{f}(n) e^{i n \theta} .
\end{aligned}
$$

Recall that $H$ satisfies the following representation formula on $\partial C$ :

$$
H=-\mathcal{S}_{\Omega}^{(0)}\left[g_{\mathrm{ele}}\right]+\mathcal{D}_{\Omega}^{(0)}\left[f_{0}\right]
$$

where $g_{\text {ele }}=\left.\frac{\partial u}{\partial \nu}\right|_{\partial \Omega}$ and $f_{0}=\left.u\right|_{\partial \Omega}$. We therefore get

$$
\begin{aligned}
H(\theta) & =\hat{f}_{0}(0)+\frac{1}{2} \sum_{n \in \mathbb{Z}^{*}}\left(\frac{1}{|n|} \hat{g}_{\text {ele }}(n)+\hat{f}_{0}(n)\right) R^{|n|} e^{i n \theta}, \\
\frac{\partial H}{\partial \theta}(\theta) & =\sum_{n \in \mathbb{Z}^{*}} i n \widehat{H}(n) e^{i n \theta}, \\
\frac{\partial^{2} H}{\partial r^{2}}(\theta) & =\frac{1}{R^{2}} \sum_{n \in \mathbb{Z}^{*}}|n|(|n|-1) \widehat{H}(n) e^{i n \theta} .
\end{aligned}
$$

Besides, for $f \in \mathcal{C}^{2, \eta}(\partial C)$, we have

$$
(I+\beta \mathcal{L})^{-1}[f](\theta)=\sum_{n \in \mathbb{Z}^{*}}\left(1+\beta \frac{|n|}{2 R}\right)^{-1} \hat{f}(n) e^{i n \theta}
$$

Note that $\widehat{\Psi}(n)=-\beta\left(1+\beta \frac{|n|}{2 R}\right)^{-1} \frac{|n|}{R} \widehat{H}(n)$.

We can now write the Fourier coefficients of $\Psi_{1}^{(1)}$ for $n \in \mathbb{Z}^{*}$ :

$$
\begin{aligned}
& \widehat{\Psi_{1}^{(1)}}(n)=-\beta \frac{1}{2} \frac{|n| R^{|n|-1}}{1+\beta \frac{|n|}{2 R}} \hat{w}(n)-\beta \sum_{p=-\infty}^{\infty} \hat{h}(p) \widehat{H}(n-p) \\
& \times\left((n-p) p+|n-p|(|n-p|-1)+\frac{\beta}{R} \frac{|n-p|^{2}}{2 R+\beta|n-p|}\right)\left(1+\beta \frac{|n|}{2 R}\right)^{-1} .
\end{aligned}
$$


Integral (4.4) becomes at first order

$$
\begin{aligned}
\mathcal{I}_{\epsilon}^{m, n}=\mathcal{I}_{0}^{m, n} & +\epsilon 2 \pi A \delta \beta \tilde{\gamma} \sum_{p=-\infty}^{\infty} \hat{h}(p) \widehat{H}(m+n-p)\left(1+\beta \frac{|m+n-p|}{2 R}\right)^{-1} \\
& -\epsilon 2 \pi B \delta \tilde{\gamma} \widehat{\Psi_{1}^{(1)}}(m+n),
\end{aligned}
$$

where

$$
\mathcal{I}_{\epsilon}^{m, n}=\int_{\partial C_{\epsilon}} \tilde{\gamma} \widetilde{c_{f r}}(x) \Phi_{\mathrm{exc}}^{n}(x) \Phi_{\mathrm{exc}}^{m}(x) d s(x)
$$

and

$$
\mathcal{I}_{0}^{m, n}=\int_{\partial C} \tilde{\gamma} c_{\mathrm{flr}}(x) \Phi_{\mathrm{exc}}^{n}(x) \Phi_{\mathrm{exc}}^{m}(x) d s(x) .
$$

4.2.3. Reconstruction of $h$. We introduce the linear operator $\mathcal{Q}$ defined on $\mathcal{C}^{2}(\partial C)$ by

$$
(\mathcal{Q}[\hat{h}])_{m, n}=\epsilon \sum_{p=-\infty}^{\infty} F_{m, n}(p) \hat{h}(p),
$$

where

$$
\begin{aligned}
F_{m, n}(p)=2 & \pi \delta \beta \tilde{\gamma}\left[\frac{A}{1+\beta \frac{|m+n-p|}{2 R}}+\frac{B}{1+\beta \frac{|m+n|}{2 R}}((m+n-p) p\right. \\
& \left.\left.+|m+n-p|(|m+n-p|-1)+\frac{\beta}{R} \frac{|m+n-p|^{2}}{2 R+\beta|m+n-p|}\right)\right] \widehat{H}(m+n-p) .
\end{aligned}
$$

Recall that $\mathcal{I}_{\epsilon}^{m, n}$ and $\mathcal{I}_{0}^{m, n}$ can be computed from the knowledge of the outgoing light intensities $I_{\mathrm{emt}, \epsilon}^{n}$ and $I_{\mathrm{emt}}^{n}$ measured at the boundary of our domain (4.2), (4.3):

$$
\mathcal{I}_{\epsilon}^{m, n}=2 \pi E_{m} \widehat{I_{\mathrm{emt}, \epsilon}^{n}}(m), \quad \mathcal{I}_{0}^{m, n}=2 \pi E_{m} \widehat{I_{\mathrm{emt}}^{n}}(m) .
$$

We denote $\hat{a}$ as the data of our problem:

$\forall m, n \in \mathbb{Z}, \quad \hat{a}_{m, n}:=2 \pi E_{m}\left(\widehat{I_{\mathrm{emt}, \epsilon}^{n}}(m)-\widehat{I_{\mathrm{emt}}^{n}}(m)\right)-\epsilon \tilde{\gamma} B \beta \pi \delta \frac{|m+n| R^{|m+n|-1}}{1+\beta \frac{|m+n|}{2 R}} \hat{w}(m+n)$,

where $\epsilon w$ is the measured difference of the voltage potential on $\partial \Omega$ when the cell occupies $C_{\epsilon}$ and when it is the circle $C$.

The operator $\mathcal{Q}$ links the perturbation $h$ of the membrane cell to the data of our problem:

$$
\hat{a}_{m, n}=(\mathcal{Q}[\hat{h}])_{m, n}+\epsilon^{2} \hat{V}_{m, n},
$$

with the term $\epsilon^{2} \hat{V}_{m, n}$ modeling the linearization error.

We choose to apply at the boundary of our domain $\Omega$ an electric field $g_{\text {ele }}: \theta \rightarrow e^{i z \theta}$ with $z \in \mathbb{Z}$. Let us compute the resulting voltage potential at the boundary of $\Omega, f_{0}$, and more specifically its Fourier coefficients. From the representation formula (3.10) of $u$ 
and the jump relations of the single and double layer potentials, we obtain the following equation at the boundary of our domain:

$$
f_{0}=-\mathcal{S}_{\Omega}^{(0)}\left[g_{\mathrm{ele}}\right]+\frac{1}{2} f_{0}+\mathcal{K}_{\Omega}^{(0)}\left[f_{0}\right]+\mathcal{D}_{C}^{(0)}[\Psi] .
$$

Since $\int_{\partial \Omega} f_{0}=0$ from (2.4), we immediately get $\hat{f}_{0}(0)=0$ and $\mathcal{K}_{\Omega}^{(0)}\left[f_{0}\right]=0$. We write, like in the previous section, the Fourier coefficients of the various layer potentials and of $\Psi$, and we get for $n \in \mathbb{Z}^{*}$

$$
\hat{f}_{0}(n)=\frac{2\left(1+\beta \frac{|n|}{2 R}\right)+\beta|n| R^{2|n|-2}}{2\left(1+\beta \frac{|n|}{2 R}\right)-\beta|n| R^{2|n|-2}} \frac{1}{|n|} \hat{g}_{\text {ele }}(n) .
$$

Note that $\hat{g}_{\text {ele }}(n)=\delta_{z}(n)$. We can now write the Fourier coefficients of $\left.H\right|_{\partial C}$ in our case:

$$
\widehat{H}(0)=0, \quad \text { and } \quad \forall n \in \mathbb{Z} \backslash\{0\}, \widehat{H}(n)=\frac{2\left(1+\beta \frac{|n|}{2 R}\right)}{2\left(1+\beta \frac{|n|}{2 R}\right)-\beta|n| R^{2|n|-2}} \frac{1}{|n|} \delta_{z}(n) R^{|z|} .
$$

The operator $\mathcal{Q}$ has therefore the following simplified expression:

$$
(\mathcal{Q}[\hat{h}])_{m, n}=\epsilon F_{m, n}(z) \hat{h}(m+n-z),
$$

where

$$
\begin{aligned}
& F_{m, n}(z)=\left[\frac{A}{1+\beta \frac{|z|}{2 R}}+\frac{B}{1+\beta \frac{|m+n|}{2 R}}\left((m+n-z) z+|z|(|z|-1)+\frac{\beta}{R} \frac{|z|^{2}}{2 R+\beta|z|}\right)\right] \\
& \times \frac{2 \pi \delta \beta \tilde{\gamma}}{|z|} \frac{2\left(1+\beta \frac{|z|}{2 R}\right)}{2\left(1+\beta \frac{|z|}{2 R}\right)-\beta|z| R^{2|z|-2}} R^{|z|} .
\end{aligned}
$$

Recall that the constants $A$ and $B$ depend on $R$ and $k$.

The adjoint of the operator $\mathcal{Q}$ is given by

$$
\left(\mathcal{Q}^{\star}[\hat{a}]\right)_{p}=\epsilon \sum_{j=-\infty}^{\infty} \bar{F}_{j, p+z-j}(z) \hat{a}_{j, p+z-j} .
$$

Then we obtain that

$$
\left(\mathcal{Q}^{\star} \mathcal{Q}[\hat{h}]\right)_{p}=\epsilon^{2} \sum_{j=-\infty}^{\infty}\left|F_{j, p+z-j}(z)\right|^{2} \hat{h}(p) .
$$

We now consider the presence of measurement or instrument noise in our measured data. We thus introduce

$$
\hat{a}_{m, n}^{\text {meas }}=(\mathcal{Q}[\hat{h}])_{m, n}+\epsilon^{2} \hat{V}_{m, n}+\sigma \hat{W}_{m, n},
$$

with the noise term $\hat{W}_{m, n}$ modeled as independent standard complex circularly symmetric Gaussian random variables (such that $\mathbb{E}\left[\left|\hat{W}_{m, n}\right|^{2}\right]=1 ; \mathbb{E}$ being the expectation). Here, 
$\sigma$ corresponds to the noise magnitude. We consider that $\sigma$ verifies $\epsilon^{2} \ll \sigma$, so that the linearization error is negligible over the measurement error and we can write

$$
\hat{a}_{m, n}^{\text {meas }}=(\mathcal{Q}[\hat{h}])_{m, n}+\sigma \hat{W}_{m, n} .
$$

Following the methodology of $[\underline{3}, 6]$, we want to assess the resolving power of the measured data in the presence of this noise.

Since $h$ is $\mathcal{C}^{2},|\hat{h}(p)| \leq C / p^{2}$ for some constant $C$, for all $p \in \mathbb{Z}^{*}$. Besides, one can see that for all $m, n \in \mathbb{Z}, F_{m, n}$ is bounded for given $R$ and $k$. Let $M$ be a positive real such that $M \ll 1 / \epsilon^{2}$. We can reconstruct the Fourier coefficients of the shape deformation $h$ only for $p$ such that $|p| \leq M$; otherwise, the linearization error $\epsilon^{2} \hat{V}_{m, n}$ is too large. We suppose that $\hat{h}_{p}=0$ for all $|p| \geq M$.

To reconstruct $h$, one can minimize the following quadratic functional over $\varphi$ :

$$
\left\|\mathcal{Q}[\hat{\varphi}]-\hat{a}^{\text {meas }}\right\|_{F}^{2},
$$

where $\hat{a}^{\text {meas }}=\left(\hat{a}_{m, n}^{\text {meas }}\right)_{m, n}, \hat{\varphi}=(\hat{\varphi}(p))_{p}$, and \|\|$_{F}$ is the Frobenius norm. The obtained least squares estimate is given by

$$
\forall p \in[-M, M], \quad \hat{h}_{e s t}(p)=\left(\mathcal{Q}^{\star} \mathcal{Q}\right)^{-1} \mathcal{Q}^{\star}\left[\hat{a}^{\text {meas }}\right](p)=\hat{h}(p)+\sigma\left(\left(\mathcal{Q}^{\star} \mathcal{Q}\right)^{-1} \mathcal{Q}^{\star}[\hat{W}]\right)_{p} .
$$

One can prove with the explicit formulas of the operators $\mathcal{Q}$ and $\mathcal{Q}^{\star}$ that the following result holds.

Proposition 4.12. Estimation (4.53) is unbiased and has the following variance:

$$
\mathbb{E}\left(\left|\hat{h}_{e s t}(p)-\hat{h}(p)\right|^{2}\right)=\frac{\sigma^{2}}{\epsilon^{2}}\left(\sum_{j=-\infty}^{\infty}\left|F_{j, p+z-j}\right|^{2}\right)^{-1} .
$$

Besides Proposition 4.12, Parseval's identity and Graf's addition formula yield

$$
\sum_{j=-\infty}^{\infty}\left|F_{j, p+z-j}\right|^{2}=\frac{2}{\pi} \int_{0}^{\pi / 2}\left|f_{p}(\theta)\right|^{2} d \theta
$$

where the function $f_{p}$ is defined by

$$
\begin{aligned}
& f_{p}(\theta)=a 2 i k R \sin (\theta) J_{p+z}^{\prime}(2 i k R \sin (\theta))+(a+R b) J_{p+z}(2 i k R \sin (\theta)), \\
& \text { with } a(R, z)=\frac{2 R^{|z|}}{2\left(1+\beta \frac{|z|}{2 R}\right)-\beta|z| R^{2|z|-2}} \frac{2 \pi \delta \beta \tilde{\gamma}}{|z|}, \\
& \qquad b(R, p, z)=a(R, z) \frac{2 R+\beta|p+z|}{2 R+\beta|z|}\left(p z+|z|(|z|-1)+\frac{\beta}{R} \frac{|z|^{2}}{2 R+\beta|z|}\right) .
\end{aligned}
$$

We introduce the signal-to-noise ratio SNR:

$$
\mathrm{SNR}=\left(\frac{\epsilon}{\sigma}\right)^{2} .
$$

The following result holds thanks to (4.54). 
Theorem 4.13. Suppose that the $p$ th mode of $h, \hat{h}(p)$, is of order 1 ; then we can resolve it if the following condition is satisfied:

$$
\mathrm{SNR}^{-1}<\frac{2}{\pi} \int_{0}^{\pi / 2}\left|f_{p}(\theta)\right|^{2} d \theta
$$

Let us simplify this stability condition under the respective asymptotic assumptions $|k| R \gg 1$ and $|k| R \ll 1$.

Since $J_{-n}=(-1)^{n} J_{n}$ ([1, Formula 9.1.5]), we can consider without any restriction that $p+z \geq 0$.

Assumption 1: $|\mathbf{k}| \mathbf{R} \gg \mathbf{1}$. We assume in this paragraph that $|k| R \gg 1$. We use the asymptotic expansions of the Bessel functions of the first kind and their derivative ([1, Formulas 9.2.5 and 9.2.11]) to find that, in this case, when $p+z<2|k| R$, we have

$$
\frac{2}{\pi} \int_{0}^{\pi / 2}\left|f_{p}(\theta)\right|^{2} d \theta \sim \frac{4 a^{2}}{\pi^{2}}|k| R \sum_{n=0}^{\infty} \frac{(4 \operatorname{Im}(i k) R)^{2 n}}{(2 n) !} \frac{2^{2 n}(n !)^{2}}{(2 n+1) !} .
$$

Then the resolving condition becomes

$$
\mathrm{SNR}^{-1}<C(R, z)|k| \quad \text { with } \quad C(R, z)=\frac{4 a(R, z)^{2}}{\pi^{2}} R \sum_{n=0}^{\infty} \frac{(4 \operatorname{Im}(i k) R)^{2 n}}{(2 n) !} \frac{2^{2 n}(n !)^{2}}{(2 n+1) !} .
$$

With large $|k| R$, we can estimate the coefficients $\hat{h}(p)$ for all SNR of order $1 /|k|$, as long as $p+z<2|k| R$.

When $p+z>2|k| R$, from [1, Formulas (9.3.35) and (9.3.43)], it follows that the following asymptotic behavior of our integrand holds:

$$
\left|f_{p}(\theta)\right|^{2} \sim \frac{\sqrt{|1-x|}}{2(p+z) \pi}|1+\sqrt{1-x}|^{-(p+z)} \mathrm{e}^{2(p+z) \operatorname{Re}(\sqrt{1-x})} x^{2(p+z-1)},
$$

where $x=\left(\frac{2 i k R \sin (\theta)}{p+z}\right)^{2}$.

Since $|x|<1$, the last term in the preceding expression is the dominant one and makes the integral exponentially small. To resolve the $p$ th mode of $h$ in this context, we therefore need an SNR exponentially large, which is impossible in practice.

We choose for each $p<M$ an electric model with $z<2|k| R-p$. The condition $p+z<2|k| R$ is in this way always satisfied, and the $p$ th mode can be resolved as long as $\mathrm{SNR}^{-1}<C(R, z)|k|$.

For a fixed $z, k$ and SNR, this inequality gives us a constraint on the cell radius. In order to be able to image the cell with a given SNR, its radius has to be larger than a minimal value $R^{\star}$, given by

$$
R^{\star}(\mathrm{SNR})=\mathcal{F}^{-1}\left(\mathrm{SNR}^{-1}\right)
$$

with

$$
\mathcal{F}(t)=\frac{4 a(t, z)^{2}}{\pi^{2}} t|k| \sum_{n=0}^{\infty} \frac{(4 R e(k) t)^{2 n}}{(2 n) !} \frac{2^{2 n}(n !)^{2}}{(2 n+1) !} .
$$

The typical size of a eukaryotes cell is $10 / 100 \mu \mathrm{m}$. We use for our different parameters the following realistic values reported in [15, [13, [16], 18]: 
- the absorption coefficient $\mu=0.03$,

- the reduced scattering coefficient $\mu_{s}^{\prime}=0.275$,

- the fluorophore quantum efficiency $\eta=0.016$,

- the fluorophore fluorescence lifetime $\tau=0.56 \mathrm{~s}^{-1}$,

- the fluorophore extinction coefficient $\varepsilon_{\text {exc }}=5 * 10^{4} \mathrm{~mm}^{-1} \mathrm{~mol}^{-1}$,

- the constant $\delta$ defined in (2.5) is given by $\delta=0.91 * 10^{-6} \mathrm{~mol} \mathrm{~V}^{-1}$.

It is worth mentioning that the absorption coefficient $\mu$ is low compared to the reduced scattering coefficient $\mu_{s}^{\prime}$. Recall that $k=\left(\frac{\mu+i \omega / c}{D}\right)^{1 / 2}$. Then, for given absorption and reduced scattering coefficients, Assumption 1 corresponds to frequencies $\omega$ such that $\omega \gg 10^{16}$ and, therefore, are nonphysical. The minimal radius $R^{\star}$ increases with $z$; we thus choose $z$ such that $|z|=1$. Since $M \sim 10$ with these values of the parameters, this choice does not impose any restriction, because we have always $M-1<2|k| R$.

Assumption 2: $|k| R \ll 1$. Note that the larger the reduced scattering coefficient is, the smaller $|k|$ is. The asymptotic expansions of the Bessel functions of the first kind and their derivative when the argument tends to zero ([1, Formula 9.1.7]) give us the asymptotic behavior of our integral in the case of a small $|k| R$ :

$$
\frac{2}{\pi} \int_{0}^{\pi / 2}\left|f_{p}(\theta)\right|^{2} d \theta \sim\left(\frac{|k| R}{2}\right)^{2(p+z)} \frac{(2(p+z)) !}{(p+z) !^{4}}(a(p+z+1)+R b)^{2} .
$$

For fixed $z, k$ and $R$, the $p$ th mode of $h$ can be resolved under Assumption 2 as long as the SNR verifies:

$$
\mathrm{SNR}^{-1}<\left(\frac{|k| R}{2}\right)^{2(p+z)} \frac{(2(p+z)) !}{(p+z) !^{4}}(a(R, z)(p+z+1)+R b(R, p, z))^{2} .
$$

If we consider now that the SNR, $k$ and $z$ are given, we can define for each mode $p$ the minimal resolving radius $R^{\star}$, i.e., the smallest radius that the cell can have if we want to resolve the $p$ th mode of its membrane deformation.

Theorem 4.14. The minimal resolving radius $R^{\star}$ has the following expression:

$$
R^{\star}(\mathrm{SNR}, p)=\mathcal{F}_{p}^{-1}\left(\mathrm{SNR}^{-1}\right),
$$

where the function $\mathcal{F}_{p}$ in this regime is given by

$$
\mathcal{F}_{p}(t)=\left(\frac{|k| t}{2}\right)^{2(p+z)} \frac{(2(p+z)) !}{(p+z) !^{4}}(a(t, z)(p+z+1)+t b(t, p, z))^{2} .
$$

Note that the higher the reduced scattering coefficient is, the better the resolving power of the imaging method is. In fact, in order to resolve the mode $p$, the higher the reduced scattering coefficient is, the smaller the required SNR is.

We plot in Figure 4.1 this minimal resolving radius as a function of the SNR for $p=0$, 1,2 and 3 . We centered the $y$-axis on the typical radii of eukaryotes cells, like in the preceding paragraph. Assumption 2 corresponds to frequencies $\omega$ such that $\omega \ll 10^{13}$. We choose $\omega=10^{9}$, which is a typical frequency used in cellular tomography. For each $p$, we took $z=\delta_{0}(p)-p$, because $R^{\star}$ decreases with $p+z$. Since we cannot take $z=0$, 


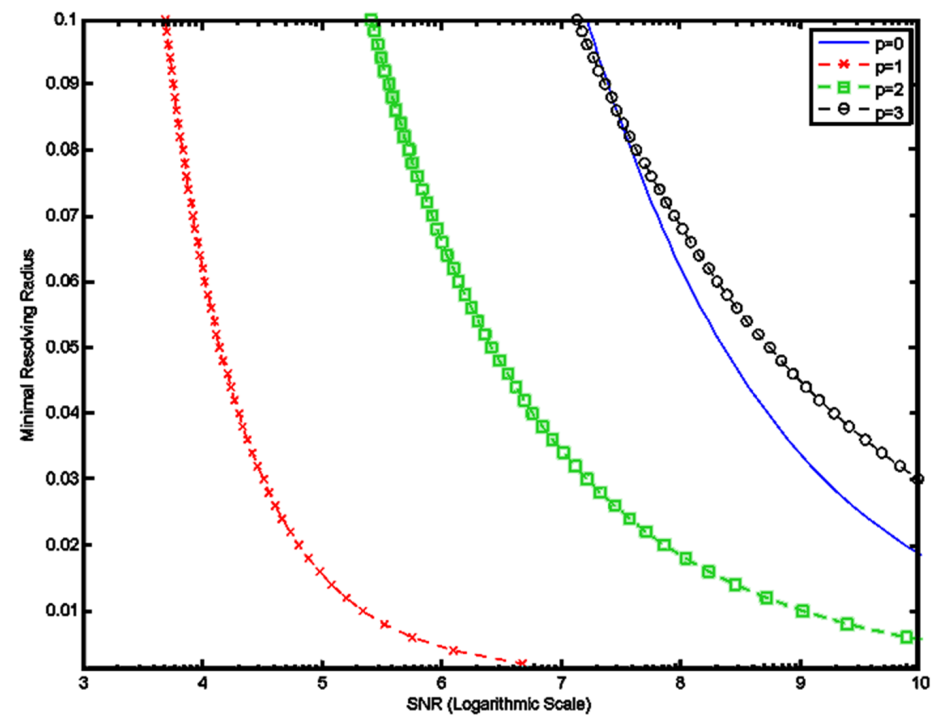

FIG. 4.1. Minimal resolving radius as function of the SNR when $|k| R \ll 1$.

the mode 0 is not the easiest to resolve. For the other parameters, we kept the values of the previous paragraph.

Under Assumption 1 for given $z, R$ and SNR, if the resolving condition was verified, we could resolve all modes of $h$ up to $M$. Because the constraint depends this time on $p$, a new question arises: "how many modes can we resolve for fixed $R$ and SNR?". We introduce the maximal mode number $p(R, \mathrm{SNR})$ defined by

$$
p(R, \mathrm{SNR})=\sup \left\{p^{\prime} \in \mathbb{N} \backslash\{0\} \mid \inf _{1 \geq p^{\prime} \geq p} \mathcal{F}_{p^{\prime}}(R)>\mathrm{SNR}^{-1}\right\}+\mathbb{1}_{\mathcal{F}_{0}(R)>\mathrm{SNR}^{-1}},
$$

which answers this question.

We plot in Figure 4.2 the maximal mode number as a function of the cell radius for different values of the SNR. We took the same values of our parameters as in Figure 4.1.

4.3. Reconstruction of the cell membrane in the general two-dimensional case. We leave the specific case of a circular domain to go back to the general case in dimension two. Let $a, b \in \mathbb{R}$ with $a<b$. Let $x:[a, b] \rightarrow \mathbb{R}^{2}$ be a parametrization of $\partial C$ such that $x \in \mathcal{C}^{2, \eta}(\mathbb{R})$ for an $\eta>0$ and $\left|x^{\prime}\right|=1$. The outward unit normal to $\partial C$ at $x(t), \nu(x)$, and the tangential vector, $T(x)$, are given by

$$
\nu(x)=R_{-\frac{\pi}{2}} x^{\prime}(t), \quad T(x)=x^{\prime}(t),
$$

where $R_{-\frac{\pi}{2}}$ is rotation by $-\frac{\pi}{2}$.

We introduce the curvature $\tau$ defined for all $x \in \partial C$ by

$$
x^{\prime \prime}(t)=\tau(x) \nu(x) .
$$

Let $C_{\epsilon}$ be an $\epsilon$-perturbation of $C$; i.e., there is $h \in \mathcal{C}^{2}([a, b])$ such that $\partial C_{\epsilon}$ is given by

$$
\partial C_{\epsilon}=\{\tilde{x} ; \tilde{x}(t)=x(t)+\epsilon h(t) \nu(x(t)), t \in[a, b]\} .
$$




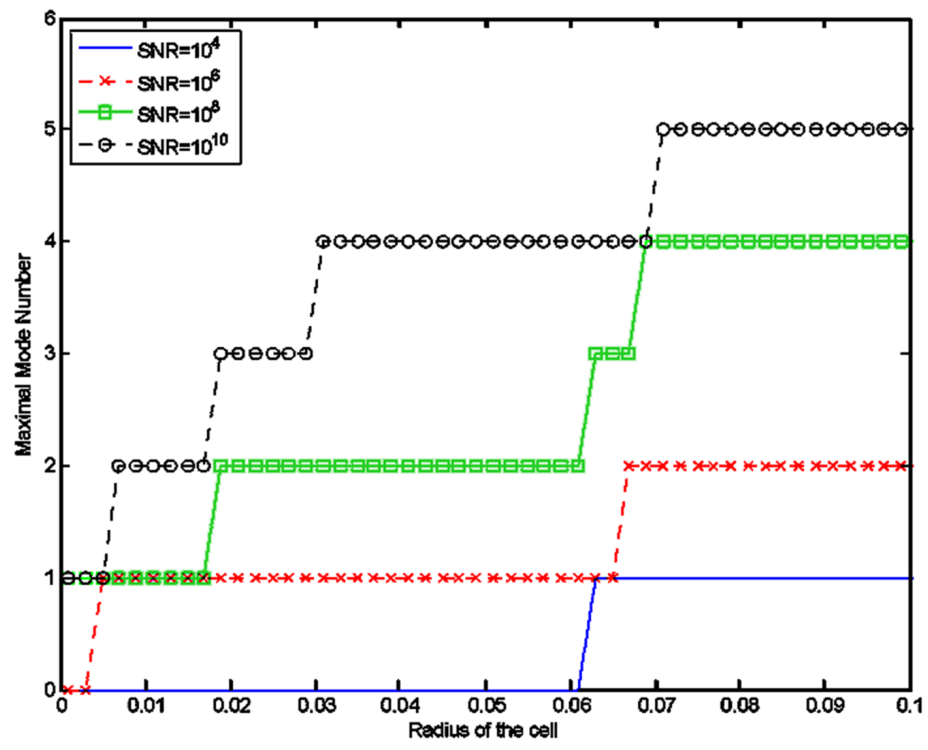

Fig. 4.2. Maximal Mode Number as function of the cell radius when $|k| R \ll 1$.

Like in the previous section, our goal is to reconstruct the shape deformation $h$ of our cell. Let $I_{\mathrm{emt}, \epsilon}^{g}$ (resp. $I_{\mathrm{emt}}^{g}$ ) be the outgoing light intensities measured at the boundary of our domain when the cell occupies $C_{\epsilon}$ (resp. $C$ ) and the optical source $g$ is applied at $\partial \Omega$. It follows from Proposition 4.1 that

$$
\begin{aligned}
\int_{\partial C_{\epsilon}} \tilde{\gamma} \widetilde{c_{\mathrm{flr}}}(x) \Phi_{\mathrm{exc}}^{f}(x) \Phi_{\mathrm{exc}}^{g}(x) d s(x) & =\int_{\partial \Omega} f I_{\mathrm{emt}, \epsilon}^{g} d s(x), \\
\operatorname{resp.} \quad \int_{\partial C} \tilde{\gamma} c_{\mathrm{flr}}(x) \Phi_{\mathrm{exc}}^{f}(x) \Phi_{\mathrm{exc}}^{g}(x) d s(x) & =\int_{\partial \Omega} f I_{\mathrm{emt}}^{g} d s(x),
\end{aligned}
$$

where $f, g \in L^{2}(\partial \Omega)$ and $\widetilde{c_{\mathrm{flr}}}$ (resp. $\left.c_{\mathrm{ffr}}\right)$ is the concentration of fluorophores on the boundary of the cell $\partial C_{\epsilon}$ (resp. $\partial C$ ).

We introduce the voltage potential $u$ such that $c_{\mathrm{flr}}=\left.\delta[u]\right|_{\partial C}$. We know from Proposition 3.5 that $u$ admits the following representation formula:

$$
\forall x \in \Omega, \quad u(x)=H(x)+\mathcal{D}_{C}^{(0)}[\Psi](x),
$$

where the harmonic function $H$ is given by

$$
\forall x \in \mathbb{R}^{2} \backslash \partial \Omega, \quad H(x)=-\mathcal{S}_{\Omega}^{(0)}\left[g_{\mathrm{ele}}\right](x)+\mathcal{D}_{\Omega}^{(0)}\left[\left.u\right|_{\partial \Omega}\right](x),
$$

and $\Psi \in \mathcal{C}^{2, \eta}(\partial C)$ satisfies the integral equation

$$
\Psi+\beta \frac{\partial \mathcal{D}_{C}^{(0)}[\Psi]}{\partial \nu}=-\beta \frac{\partial H}{\partial \nu} \quad \text { on } \partial C \text {. }
$$


We compute the first-order approximation of $\widetilde{c_{\mathrm{fr}}}$ using exactly the same method as in Subsection 4.2. Doing so, we arrive with the help of Corollary 4.8 at

$$
\widetilde{c_{\mathrm{flr}}}=c_{\mathrm{flr}}-\epsilon \delta \Psi_{1}^{(1)}+o(\epsilon)
$$

where the function $\Psi_{1}^{(1)}$ is defined by

$$
\begin{aligned}
\Psi_{1}^{(1)} & =-\beta(I+\beta \mathcal{L})^{-1}\left(-\tau h^{\prime} \frac{\partial H}{\partial T}+h \frac{\partial^{2} H}{\partial \nu^{2}}+\frac{\partial}{\partial \nu} \mathcal{D}_{\Omega}^{(0)}[w]-h \frac{\partial}{\partial \nu} \mathcal{D}_{C}^{(0)}[\Psi]\right. \\
& \left.+\frac{\partial}{\partial T} \mathcal{K}_{C}^{(0)}\left[h \frac{\partial \Psi}{\partial T}\right]-\frac{\partial}{\partial T} h \frac{\partial}{\partial T} \mathcal{K}_{C}^{(0)}[\Psi]\right)
\end{aligned}
$$

and $w$ is the solution to the problem

$$
\begin{cases}\Delta w=0 & \text { in } C \cup \Omega \backslash \bar{C}, \\ \left.\frac{\partial w}{\partial \nu}\right|_{+}-\left.\frac{\partial w}{\partial \nu}\right|_{-}=-\frac{\partial}{\partial T} h \frac{\partial \Psi}{\partial T} & \text { on } \partial C, \\ \left.w\right|_{+}-\left.w\right|_{-}-\left.\beta \frac{\partial w}{\partial \nu}\right|_{-}=-\beta\left(\tau h \frac{\partial u}{\partial \nu}+\left.\frac{\partial}{\partial T} h \frac{\partial u}{\partial T}\right|_{-}\right) & \text {on } \partial C, \\ \left.\frac{\partial w}{\partial \nu}\right|_{\partial \Omega}=0 & \text { on } \partial \Omega .\end{cases}
$$

We then obtain an expansion of (4.56) as $\epsilon \rightarrow 0$.

Proposition 4.15. Integral (4.56) admits at first order in $\epsilon$ the following expansion:

$$
\begin{gathered}
\int_{\partial C_{\epsilon}} \tilde{\gamma} \widetilde{c_{\mathrm{flr}}}(x) \Phi_{\mathrm{exc}}^{f}(x) \Phi_{\mathrm{exc}}^{g}(x) d s(x)=\int_{\partial C} \tilde{\gamma} c_{\mathrm{ffr}}(x) \Phi_{\mathrm{exc}}^{f}(x) \Phi_{\mathrm{exc}}^{g}(x) d s(x) \\
+\epsilon \int_{a}^{b} \tilde{\gamma}\left(A(t) c_{\mathrm{flr}}(t) h(t)-\delta B(t) \Psi_{1}^{(1)}(t)\right) d t+o(\epsilon),
\end{gathered}
$$

where the functions $A$ and $B$ are given by

$$
\begin{aligned}
& A=\frac{d \Phi_{\mathrm{exc}}^{f}(t)}{d t} \Phi_{\mathrm{exc}}^{g}(t)+\Phi_{\mathrm{exc}}^{f}(t) \frac{d \Phi_{\mathrm{exc}}^{g}(t)}{d t}-\tau(t) \Phi_{\mathrm{exc}}^{f}(t) \Phi_{\mathrm{exc}}^{g}(t), \\
& B=\Phi_{\mathrm{exc}}^{f}(t) \Phi_{\mathrm{exc}}^{g}(t) .
\end{aligned}
$$

Let $f_{1}, \ldots, f_{L}$, be a finite number of linearly independent functions in $L^{2}(\partial \Omega)$. We introduce the functional $\mathcal{J}$ defined on $\mathcal{C}^{2}([a, b])$ by

$$
\mathcal{J}(h)=\sum_{i, j=1}^{L}\left|\int_{\partial \Omega} f_{i}\left(I_{\mathrm{emt}, \epsilon}^{f_{j}}-I_{\mathrm{emt}}^{f_{j}}\right) d s-\epsilon \int_{a}^{b} \tilde{\gamma}\left(A_{i, j}(t) c_{\mathrm{frr}}(t) h(t)-\delta B_{i, j}(t) \Psi_{1}^{(1)}(t)\right) d t\right|^{2},
$$

where the functions $A_{i, j}$ and $B_{i, j}$ are given by

$$
\begin{aligned}
& A_{i, j}=\frac{d \Phi_{\mathrm{exc}}^{f_{i}}(t)}{d t} \Phi_{\mathrm{exc}}^{f_{j}}(t)+\Phi_{\mathrm{exc}}^{f_{i}}(t) \frac{d \Phi_{\mathrm{exc}}^{f_{j}}(t)}{d t}-\tau(t) \Phi_{\mathrm{exc}}^{f_{i}}(t) \Phi_{\mathrm{exc}}^{f_{j}}(t), \\
& B_{i, j}=\Phi_{\mathrm{exc}}^{f_{i}}(t) \Phi_{\mathrm{exc}}^{f_{j}}(t) .
\end{aligned}
$$


We reconstruct the shape deformation $h$ by minimizing the functional $\mathcal{J}$ over $h$. In order to maximize the resolution of the reconstructed images, we choose $f_{1}, \ldots, f_{L}$ such that the functions $A_{i, j}$ and $B_{i, j}$ for $i, j \in[1, L]$ are highly oscillating. We will then be able to obtain a resolved reconstruction of the boundary changes $h$.

We introduce the operator $\Lambda: L^{2}(\partial \Omega) \rightarrow L^{2}(\partial C)$ defined by

$$
\forall f \in L^{2}(\partial \Omega), \forall z \in \partial C, \quad \Lambda[f](z)=\left.\Phi_{\operatorname{exc}}^{f}\right|_{\partial C}(z)=\int_{\partial \Omega} G_{z}(y) f(y) d s(y) .
$$

The adjoint operator $\Lambda^{\star}: L^{2}(\partial C) \rightarrow L^{2}(\partial \Omega)$ is given by

$$
\forall q \in L^{2}(\partial C), \forall y \in \partial \Omega, \quad \Lambda^{\star}[q](y)=\left.p\right|_{\partial \Omega}(y)=\int_{\partial C} \overline{G_{z}(y)} q(z) d s(z),
$$

where $p$ is the solution to the problem

$$
\begin{cases}-\Delta p+k^{2} p=0 & \text { in } \Omega, \\ \left.\frac{\partial p}{\partial \nu}\right|_{+}-\left.\frac{\partial p}{\partial \nu}\right|_{-}=-q & \text { on } \partial C, \\ \left.p\right|_{+}-\left.p\right|_{-}=0 & \text { on } \partial C, \\ \ell \frac{\partial p}{\partial \nu}+p=0 & \text { on } \partial \Omega .\end{cases}
$$

We therefore obtain the following expression for $\Lambda^{\star} \Lambda$ :

$$
\forall f \in L^{2}(\partial \Omega), \forall y \in \partial \Omega, \quad \Lambda^{\star} \Lambda[f](y)=\int_{\partial \Omega} d t f(t) \int_{\partial C} \overline{G_{z}(y)} G_{z}(t) d s(z) .
$$

Following [2,4], we choose $f_{1}, \ldots, f_{L}$ to be the first singular vectors of the operator $\Lambda$. The number $L$, which fixes the resolving power of the approach, is chosen to maximize the trade-off between resolution and stability. To gain resolution, one has to choose $L$ as large as possible. But if it is too large, then it follows from the fact that $f_{i}$ is highly oscillating for large $i$ that the algorithm is unstable in the case of noisy data [4,5].

5. Conclusion. In this paper we have introduced and analyzed a mathematical model for optical imaging of cell membrane potentials changes induced by applied currents. We have presented a direct imaging algorithm in the linearized case and provided explicit formulas for its resolving power of the measurements in the presence of measurement noise. We have suggested an iterative algorithm for complex shapes. It would be interesting to consider the case of cluttered cells. Another challenging problem is the tracking of membrane changes in cell mechanisms such as cell division. This would be the subject of a forthcoming work.

Appendix A. Explicit calculation of $G_{z}$ in the case of a sphere. We consider in this appendix that the dimension is three and that $\Omega$ is the unit sphere. We expand $G$, the solution to (3.3), in spherical harmonics $\left(Y_{m}^{l}\right)$ :

$$
\forall z \in \Omega, \forall y(1, \theta, \phi) \in \partial \Omega, \quad G_{z}(y)=\sum_{l=0}^{\infty} \sum_{m=-l}^{l} g_{m}^{l, z} Y_{m}^{l}(\theta, \phi) .
$$


An addition theorem [1, Formula (10-1-45/46)] gives us the expansion of $\Gamma$ :

$$
\forall z\left(r^{\prime}, \theta^{\prime}, \phi^{\prime}\right) \in \Omega, \forall y \in \partial \Omega, \quad \Gamma_{z}(y)=i k \sum_{l=0}^{\infty} \sum_{m=-l}^{l} j_{l}\left(i k r^{\prime}\right) h_{l}^{(1)}(i k) Y_{m}^{l}\left(\theta^{\prime}, \phi^{\prime}\right) Y_{m}^{l}(\theta, \phi),
$$

where $j_{l}$ and $h_{l}^{(1)}$ are respectively the spherical Bessel and Hankel functions of first kind of order $l$.

We then express the operators $\mathcal{S}_{\Omega}$ and $\mathcal{K}_{\Omega}$ in terms of spherical harmonics [27, in the same way we wrote their Fourier coefficients in the previous section:

$$
\begin{aligned}
& \forall y \in \partial \Omega, \quad\left(-\frac{I}{2}+\mathcal{K}_{\Omega}\right)[q](y)=-\sum_{l=0}^{\infty} \sum_{m=-l}^{l} k^{2} j_{l}^{\prime}(i k) h_{l}^{(1)}(i k) q_{m}^{l} Y_{m}^{l}(\theta, \phi), \\
& \forall y \in \partial \Omega, \quad \mathcal{S}_{\Omega}[q](y) \quad=i \sum_{l=0}^{\infty} \sum_{m=-l}^{l} k j_{l}(i k) h_{l}^{(1)}(i k) q_{m}^{l} Y_{m}^{l}(\theta, \phi),
\end{aligned}
$$

for

$$
\forall y(1, \theta, \phi) \in \partial \Omega, \quad q(y)=\sum_{l=0}^{\infty} \sum_{m=-l}^{l} q_{m}^{l} Y_{m}^{l}(\theta, \phi) .
$$

From (3.5) we obtain

$$
g_{m}^{l, z}=\frac{i k j_{l}\left(i k r^{\prime}\right) h_{l}^{(1)}(i k) Y_{m}^{l}\left(\theta^{\prime}, \phi^{\prime}\right)}{-k^{2} j_{l}^{\prime}(k) h_{l}^{(1)}(i k)+\frac{1}{\ell} i k j_{l}(i k) h_{l}^{(1)}(i k)}=\frac{j_{l}\left(i k r^{\prime}\right)}{i k j_{l}^{\prime}(i k)+\frac{1}{\ell} j_{l}(i k)} Y_{m}^{l}\left(\theta^{\prime}, \phi^{\prime}\right),
$$

or else, for all $z=\left(r^{\prime}, \theta^{\prime}, \phi^{\prime}\right) \in \Omega$ and $y=(1, \theta, \phi) \in \partial \Omega$,

$$
G_{z}(y)=\sum_{l=0}^{\infty} \sum_{m=-l}^{l} \frac{j_{l}\left(i k r^{\prime}\right)}{i k j_{l}^{\prime}(i k)+\frac{1}{\ell} j_{l}(i k)} Y_{m}^{l}\left(\theta^{\prime}, \phi^{\prime}\right) Y_{m}^{l}(\theta, \phi) .
$$

Note that we find a very similar formula as the one in 2D. The Bessel function of the first kind is replaced by the spherical function of the first kind, and our operator is decomposed in the spherical harmonics basis instead of the Fourier basis.

\section{REFERENCES}

[1] M. Abramowitz and I. A. Stegun, Handbook of Mathematical Functions, National Bureau of Standards, Applied Mathematics Series, Vol. 55, 1964.

[2] Habib Ammari, Elena Beretta, Elisa Francini, Hyeonbae Kang, and Mikyoung Lim, Reconstruction of small interface changes of an inclusion from modal measurements II: the elastic case, J. Math. Pures Appl. (9) 94 (2010), no. 3, 322-339, DOI 10.1016/j.matpur.2010.02.001 (English, with English and French summaries). MR2679030 (2011m:35412)

[3] Mathematical and statistical methods for imaging, Contemporary Mathematics, vol. 548, American Mathematical Society, Providence, RI, 2011. Papers from the NIMS Thematic Workshop held at Inha University, Incheon, August 10-13, 2010; Edited by Habib Ammari, Josselin Garnier, Hyeonbae Kang and Knut Sølna. MR2868483(2012h:65006)

[4] Habib Ammari, Pierre Garapon, François Jouve, Hyeonbae Kang, Mikyoung Lim, and Sanghyeon $\mathrm{Yu}$, A New Optimal Control Approach for the Reconstruction of Extended Inclusions, SIAM J. Control Optim. 51 (2013), no. 2, 1372-1394, DOI 10.1137/100808952. MR3038017

[5] Habib Ammari, Josselin Garnier, Hyeonbae Kang, Mikyoung Lim, and Knut Sølna, Multistatic imaging of extended targets, SIAM J. Imaging Sci. 5 (2012), no. 2, 564-600, DOI 10.1137/10080631X. $\operatorname{MR} 2971173$ 
[6] H. Ammari, J. Garnier, and K. Sølna, Limited view resolving power of conductivity imaging from boundary measurements, SIAM J. Math. Anal. 45 (2013), 1704-1722.

[7] Habib Ammari and Hyeonbae Kang, Reconstruction of small inhomogeneities from boundary measurements, Lecture Notes in Mathematics, vol. 1846, Springer-Verlag, Berlin, 2004. MR2168949 (2006k:35295)

[8] Habib Ammari and Hyeonbae Kang, Polarization and moment tensors, with applications to inverse problems and effective medium theory, Applied Mathematical Sciences, vol. 162, Springer, New York, 2007. MR2327884 (2009f:35339)

[9] Habib Ammari, Hyeonbae Kang, Mikyoung Lim, and Habib Zribi, Conductivity interface problems. I. Small perturbations of an interface, Trans. Amer. Math. Soc. 362 (2010), no. 5, 2435-2449, DOI 10.1090/S0002-9947-09-04842-9. MR2584606 (2012f:35077)

[10] Elena Beretta and Elisa Francini, Asymptotic formulas for perturbations in the electromagnetic fields due to the presence of thin inhomogeneities, Inverse problems: theory and applications (Cortona/Pisa, 2002), Contemp. Math., vol. 333, Amer. Math. Soc., Providence, RI, 2003, pp. 49-62, DOI 10.1090/conm/333/05953. MR2032006(2005a:35263)

[11] Elena Beretta, Elisa Francini, and Michael S. Vogelius, Asymptotic formulas for steady state voltage potentials in the presence of thin inhomogeneities. A rigorous error analysis, J. Math. Pures Appl. (9) 82 (2003), no. 10, 1277-1301, DOI 10.1016/S0021-7824(03)00081-3 (English, with English and French summaries). MR2020923(2004i:35021)

[12] David Colton and Rainer Kress, Inverse acoustic and electromagnetic scattering theory, 2nd ed., Applied Mathematical Sciences, vol. 93, Springer-Verlag, Berlin, 1998. MR1635980 (99c:35181)

[13] A. Corlu, R. Choe, T. Durduran, M. A. Rosen, M. Schweiger, S. R. Arridge, M. D. Schnall, and A. G. Yodh, Three-dimensional in vivo fluorescence diffuse optical tomography of breast cancer in humans, Optics Express, 15 (2007), 6696-6716.

[14] Marc Duruflé, Victor Péron, and Clair Poignard, Time-harmonic Maxwell equations in biological cells - the differential form formalism to treat the thin layer, Confluentes Math. 3 (2011), no. 2, 325-357, DOI 10.1142/S1793744211000345. MR2807112(2012f:35520)

[15] H. Egger, M. Freiberger, and M. Schlottbom, Analysis of forward and inverse models in fluorescence optical tomography, Aachen Institute for Advanced Study in Computational Engineering Science, November 2009.

[16] M. J. Eppstein, A.Godavarty, D. J. Hawrysz, R. Roy, and E. M. Sevick-Muraca, Influence of the refractive index-mismatch at the boundaries measured in fluorescence-enhanced frequency-domain photon migration imaging, Optics Express, 10 (2002), 653-662.

[17] R. Gowrishankar and J. C. Weaver, An approach to electrical modeling of single and multiple cells, Proc. Nat. Acad. Sci., 100 (2003), 3203-3208.

[18] D. Gross, L. M. Loew, and W. W. Webb, Optical imaging of cell membrane potential changes induced by applied electric fields, Biophysical J., 50 (1986), 339-348.

[19] C. L. Hutchinson, J. R. Lakowicz, and E. M. Sevick-Muraca, Fluorescence life-time based sensing in tissues: a computational study, Biophys. J., 68 (1995), 1574-1582.

[20] Tosio Kato, Perturbation theory for linear operators, Die Grundlehren der mathematischen Wissenschaften, Band 132, Springer-Verlag New York, Inc., New York, 1966. MR0203473 (34 \#3324)

[21] Abdessatar Khelifi and Habib Zribi, Asymptotic expansions for the voltage potentials with twodimensional and three-dimensional thin interfaces, Math. Methods Appl. Sci. 34 (2011), no. 18, 2274-2290, DOI 10.1002/mma.1529. MR2861742 (2012j:35029)

[22] Rainer Kress, Linear integral equations, 2nd ed., Applied Mathematical Sciences, vol. 82, SpringerVerlag, New York, 1999. MR.1723850 (2000h:45001)

[23] Dionisios Margetis and Nikos Savva, Low-frequency currents induced in adjacent spherical cells, J. Math. Phys. 47 (2006), no. 4, 042902, 18, DOI 10.1063/1.2190333. MR.2226331 (2006m:78018)

[24] V. A. Markel and J. C. Schotland, Inverse problem in optical diffusion tomography. II. Role of boundary conditions, J. Opt. Soc. Amer. A, 19 (2002), 558-566.

[25] V. A. Markel and J. C. Schotland, Multiple projection optical diffusion tomography with plane wave illumination, Phys. Med. Biol., 50 (2005), 2351-2364.

[26] A. B. Milstein, S. Oh, K. J. Webb, C. A. Bouman, Q. Zhang, D. A. Boas, and R. P. Millane, Fluorescence optical diffusion tomography, Applied Optics, 42 (2003), 3081-3094.

[27] Jean-Claude Nédélec, Acoustic and electromagnetic equations, integral representations for harmonic problems, Applied Mathematical Sciences, vol. 144, Springer-Verlag, New York, 2001. MR1822275 (2002c:35003) 
[28] V. Ntziachristos, Fluorescence molecular imaging, Annu. Rev. Biomed. Eng., 8 (2006), 1-33.

[29] M. A. O'Leary, D. A. Boas, X. D. Li, B. Chance, and A. G. Yodh, Fluorescence lifetime imaging in turbid media, Opt. Lett., 21 (1996), 158-160.

[30] M. S. Patterson, B. Chance, and B. C. Wilson, Time resolved reflectance and transmittance for the non-invasive measurement of tissue optical properties, Appl. Opt., 28 (1989), 2331-2336.

[31] M. S. Patterson and B. W. Pogue, Mathematical model for time resolved and frequency-domain fluorescence spectroscopy in biological tissues, Appl. Opt., 33 (1994), 1963-1974.

[32] Clair Poignard, Asymptotics for steady-state voltage potentials in a bidimensional highly contrasted medium with thin layer, Math. Methods Appl. Sci. 31 (2008), no. 4, 443-479, DOI 10.1002/mma.923. MR2387417 (2009a:35049)

[33] Clair Poignard, About the transmembrane voltage potential of a biological cell in time-harmonic regime, Mathematical methods for imaging and inverse problems, ESAIM Proc., vol. 26, EDP Sci., Les Ulis, 2009, pp. 162-179, DOI 10.1051/proc/2009012 (English, with English and French summaries). MR2498146(2010j:92046)

[34] C. Poignard, P. Dular, R. Perrussel, L. Krähenbühl, L. Nicolas, and M. Schatzman, Approximate conditions replacing thin layers, IEEE Trans. Mag., 44 (2008), 1154-1157.

[35] M. C. W. van Rossum and Th. M. Nieuwenhuizen, Multiple scattering of classical waves: microscopy, mesoscopy, and diffusion, Rev. Modern Phys., 71 (1999), 313-371.

[36] R. Roy and E. M. Sevick-Muraca, Truncated Newton's optimization schemes for absorption and fluorescence optical tomography: Part I, theory and formulation, Optics Express, 4 (1999), 353-371.

[37] E. M. Sevick and C. L. Burch, Origin of phosphorescence signals reemitted from tissues, Opt. Lett., 19 (1994), 1928-1930.

[38] John C. Schotland, Path integrals and optical tomography, Mathematical and statistical methods for imaging, Contemp. Math., vol. 548, Amer. Math. Soc., Providence, RI, 2011, pp. 77-84, DOI 10.1090/conm/548/10837. MR2868489(2012j:65307)

[39] Elias M. Stein, Singular integrals and differentiability properties of functions, Princeton Mathematical Series, No. 30, Princeton University Press, Princeton, N.J., 1970. MR0290095 (44 \#7280)

[40] D. J. Stephens and V. J. Allan, Light microscopy techniques for live cell imaging, Science, 300 (2003), 82-86. 